\title{
Gradhiva
}

GRADHIV

Revue d'anthropologie et d'histoire des arts

\section{L'expérience de Bernard Maupoil au Dahomey : entre science et engagement, un laboratoire pour l'ethnologie en milieu colonial}

Bernard Maupoil in Dahomey: Between Science and Personal Commitment, a Field of Experiment for Ethnology in a Colonial Context

\section{Valérie Perlès}

\section{OpenEdition \\ Journals}

\section{Édition électronique}

URL : https://journals.openedition.org/gradhiva/5716

DOI : 10.4000/gradhiva.5716

ISSN : 1760-849X

\section{Éditeur}

Musée du quai Branly Jacques Chirac

\section{Édition imprimée}

Date de publication : 24 mars 2021

Pagination : $192-216$

ISBN : 978-2-35744-132-3

ISSN : 0764-8928

\section{Référence électronique}

Valérie Perlès, «L'expérience de Bernard Maupoil au Dahomey : entre science et engagement, un laboratoire pour l'ethnologie en milieu colonial », Gradhiva [En ligne], 32 | 2021, mis en ligne le 02 avril 2021, consulté le 25 mai 2021. URL : http://journals.openedition.org/gradhiva/5716 ; DOI : https:// doi.org/10.4000/gradhiva.5716 
Études et essais

L'expérience de

Bernard Maupoil

au Dahomey:

entre science

et engagement,

un laboratoire

pour l'ethnologie

en milieu colonial

Mots clefs:

Dahomey, collecte, terrain colonial,

musée d'Ethnographie du Trocadéro, vodun

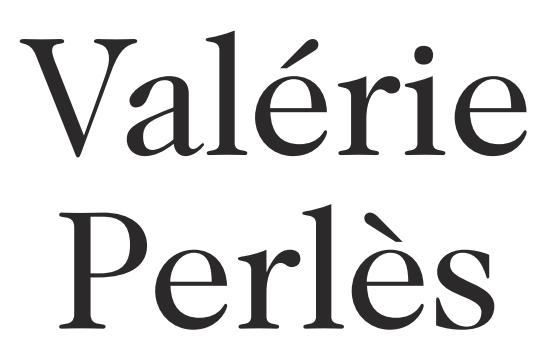


Cet article vise, dans le contexte des récents débats autour de la restitution en faveur des pays africains, à donner corps à une expérience concrète de terrain dans le Dahomey des années 1930, associant une collecte d'objets pour le musée d'Ethnographie du Trocadéro et une thèse au sein de l'Institut d'ethnologie de Paris. Le fonds de correspondance de Bernard Maupoil exploité ici révèle la dimension empirique de sa démarche, la structuration de ses réseaux, les contraintes institutionnelles, ainsi que sa relation aux populations locales et son engagement personnel, montrant que la nature des objets ramenés dépend autant de la réalité du terrain que du positionnement du chercheur.

De 1899 à 1960, le Dahomey fait partie de l'Empire français. Avant la conquête, une partie de ce territoire s'organisait en royaumes, le plus puissant étant celui du Danhomè. En 1894, après quatre années de conflit, les soldats Français soumettent le roi du Danhomè, Béhanzin, et constituent au passage un butin de guerre conservé en partie au musée du quai BranlyJacques Chirac. Ce dernier, conformément à l'engagement pris par le Président Macron à l'université de Ouagadougou le 28 novembre 2017, a fait peu après l'objet d'une démarche de restitution au Bénin $\mathbf{1}$.

Bernard Maupoil, scientifique et administrateur colonial, a donc passé deux années au Dahomey au cours desquelles il a produit une thèse au sein de I'Institut d'ethnologie et effectué une collecte d'objets pour le musée d'Ethnographie du Trocadéro $(M E T)^{2}$. Nous allons voir ici que la nature des objets qu'il a prélevés dépend autant de la réalité du terrain que de son positionnement en tant que scientifique devant composer avec ce à quoi il a accès, ce qu'il en comprend et ce qu'il veut finalement en dire. Ainsi, si l'objet appartient naturellement à la culture qui l'a créé, il est également le produit du processus ayant permis de l'en extraire et raconte, en creux, le parcours idiosyncratique du collecteur. Son immersion au cœur de la culture dahoméenne, l'approfondissement de son projet de thèse consacré à la divination vont en effet largement influencer le rapport de Maupoil à la culture matérielle.

Le parcours de l'ethnologue a pu être reconstitué avec précision grâce à un fonds de correspondances privées, mis en ligne par le Conseil international de la langue française ${ }^{3}$. Les recherches se sont ensuite poursuivies au sein de fonds mieux connus, tels celui du MET à la bibliothèque centrale du Muséum national d'histoire naturelle et la correspondance de Marcel Mauss conservée au Collège de France.
Comme l'a analysé Christophe Lebaune, ce matériau brut, constitué de fragments, «portela marque des hésitations, revirements et corrections, bref, $d$ e la recherche en train de se faire» (Labaune 2017). Au-delà du positionnement épistémologique (réalité du terrain, constitution des réseaux, contraintes institutionnelles, etc.), cette correspondance témoigne également de l'engagement personnel et des états d'âme de son auteur, utiles pour mieux comprendre le jeu des acteurs. La lecture de la thèse de Maupoil $\left(1943^{4}\right)$, restitution formalisée de l'expérience du terrain, est bien entendu venue compléter ce corpus.

\section{LES ETHNOLOGUES DE TERRAIN FACE À LA RÉALITÉ DE LA COLONISATION: ENTRE SCIENCE ET POLITIQUE}

Dans le contexte de l'après-guerre, la mise en valeur de l'Empire colonial est une obligation patriotique avec laquelle les ethnologues doivent composer. La gauche française, dont font partie les pères fondateurs de l'ethnologie, choisit d'assumer l'héritage républicain de la «mission civilisatrice» contre l'anticolonialisme de principe des communistes (Loyau 2017) La rhétorique d'une gestion éclairée, qui tiendrait compte de la culture des populations afin de mieux les administrer, est alors développée. Avec la reconnaissance progressive de l'utilité de l'érudition coloniale, source de connaissance scientifique désormais légitime, I'Institut d'ethnologie est créé par le ministère des Colonies en 1925. Paul Rivet et Marcel Mauss en prennent la direction, confirmant leur adhésion à une cause coloniale à laquelle les sciences de l'homme peuvent aisément contribuer (Conklin 2015: 25). L'un des principaux objectifs est de former des professionnels de l'enquête, élèves de l'Institut ou «travailleurs
1. «Remise du rapport Savoy/ Sarr sur la restitution du patrimoine africain» [en liǵne], Élysée.fr, 23 novembre 2018, disponible sur: https://www. elysee fr/emmanuel-macron/ elyse.fr/entman/ 2018/11/23/remise-du-rapportsavoy-sarr-sur-la-restitutiondu-patrimoine-africain (consulté le 9 décembre 2020).

2. Je remercie ici : Étienne Féau, qui m'a soutenue et conseillée dans la mise en œuvre de cette recherche suite au projet d'exposition sur le fonds «Dahomey» des Archives de la planète d'Albert Kahn; Sophie Blanchy, pour sa relecture attentive qui m'a aidée à structurer ma démonstration et Amandine Yehouetome qui, lors d'un stage au musée Albert Kahn, m'a patiemment expliqué ce qu'était le vodun.

3. En 1971, Élisabeth Maupoil rencontre Hubert Joly, président du Conseil international de la langue française et ancien administrateur de la France d'Outre-Mer. Elle décide alors de léguer ses biens au CILF et de charger cette institution de conserver la mémoire de son mari.

4. Initialement publiée en 1943 sa thèse a été soutenue en 1946 à titre posthume par Marcel Griaule, suite à son décès le 15 décembre 1944 au camp de concentration de Hersbruck. 


\section{Études et essais}

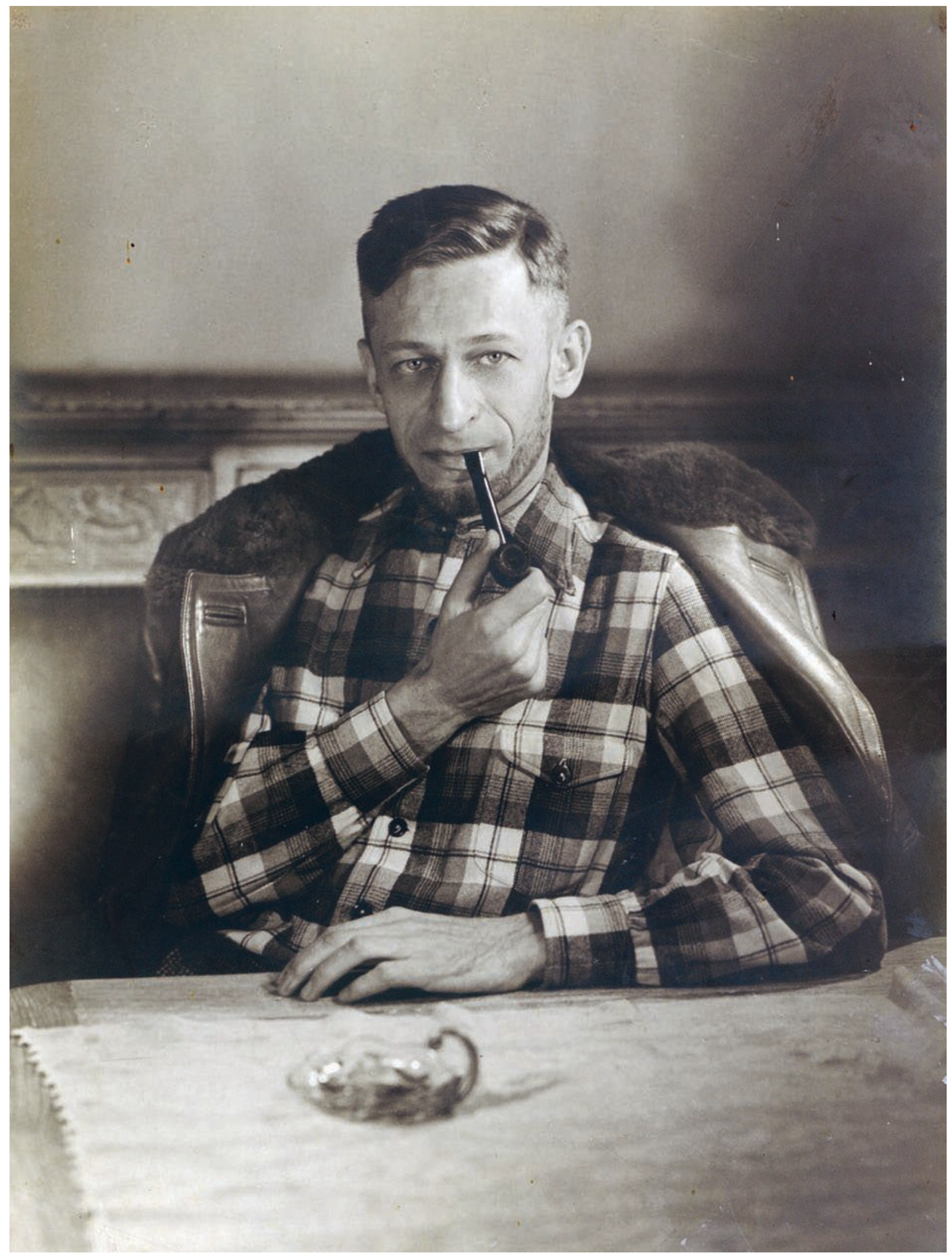

Anonyme, Portrait de Bernard Maupoil, Conseil international de la langue française. 
indigènes», et de publier leurs travaux, constituant ainsi une base méthodologique et théorique pour la nouvelle discipline.

L'étude du parcours de Maupoil, ancien stagiaire du musée du Trocadéro, va nous permettre de mieux comprendre comment les ethnologues travaillent sur leur terrain, et affrontent les contradictions qui surgissent avec leurs convictions profondes. Lorsque Maupoil annonce à Rivet qu'il souhaite quitter le MET et embrasser une carrière dans l'administration coloniale, ce dernier appuie son souhait d'aller au Dahomey plutôt qu'à Dakar, où il est d'abord affecté $\mathbf{5}$. Le gouverneur général des colonies donne son accord, reconnaissant volontiers que le Sénégal, désormais «européanisé», n'est pas le terrain le plus propice à l'ethnologie, le Dahomey apparaissant

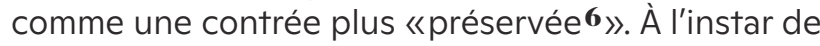
ses jeunes collègues envoyés sur le terrain, Maupoil devient un observateur de premier plan de la réalité vécue par les populations, loin des discours de propagande. Au lieu du rôle «civilisateur» annoncé, la présence coloniale lui apparaît comme une source de servitude, de corruption, de dénaturation et d'affaiblissement des liens sociaux (Conklin 2015: 43). Au fil des mois, il assume de plus en plus difficilement son implication dans un système qu'il perçoit désormais comme violent et raciste. Il est très critique à l'égard des chefs de canton, notamment ceux désignés par l'administration française après la destitution du dernier roi d'Abomey, Ago-li-Agbo, en 1900. La nomination de princes de sang à cette fonction, en parfaite contradiction avec la tradition royale dahoméenne, vise à parachever la dislocation du royaume du Danhomè ${ }^{7}$. À l'issue de son séjour, il souhaite donc livrer son opinion, «désobligeante pour la colonisation capitaliste», dans la conclusion de sa thèse ${ }^{\mathbf{8}}$. Mauss met néanmoins en garde son élève au sujet de ces allusions contre le système qui leur permet malgré tout d'exister:

\begin{abstract}
Je vous déconseille tout à fait fortement de parler de quoi que ce soit concernant vos avis sur la colonisation, et spécialement la colonisation capitaliste. Cela ne peut que diminuer la valeur scientifique de votre travail. [...] Naturellement, vous le comprenez bien, il n'y a pas l'ombre d'une opposition de principe à vos idées, mais il y a la nécessité d'une certaine discrétion?.
\end{abstract}

La plupart des ethnologues - et Mauss en fait partie - expriment plus ou moins ouvertement des réserves sur la manière dont les populations sont traitées par l'administration métropolitaine, sans toutefois la remettre radicalement en cause. Pour dépasser cette contradiction, le travail de terrain est finalement revendiqué comme une activité neutre et apolitique, la principale tâche de l'ethnologue étant de rendre compte de modes de vie traditionnels menacés par la «modernité $\mathbf{1 0}$ ».
Acteur d'une science qui a pour objet la connaissance de la culture de l'autre, l'ethnologue sur le terrain colonial est donc partagé, puisqu'il y incarne une figure de domination tout en y faisant l'expérience d'une relation privilégiée aux populations. C'est dans ce contexte favorable aux sciences humaines que se négocie également la place à donner aux élites locales, engagées elles aussi dans la production d'un discours positiviste sur leur propre culture. L'ensemble de ces éléments ont, nous allons le voir, des impacts concrets sur la politique de collecte qui se déploie sur fond de construction d'un discours de propagande coloniale et de légitimation de l'ethnologie, jeune discipline alors «bricolée» entre immersion, observation, accumulation, engagement, prise de recul, écriture, diffusion et remise en question (Laurière 2017: 447).

\section{DE «BEAUX》 OBJETS POUR PROMOUVOIR L'EMPIRE COLONIAL}

Paul Rivet, perçu comme la figure de proue de la nouvelle génération d'anthropologues, considère que l'ethnologie est la science qui peut «changer le regard porté sur les populations "primitives" et combattre les préjugés racistes» (ibid.). En 1928, après son élection à la chaire d'anthropologie du Muséum national d'histoire naturelle, il fait rattacher le MET à cette prestigieuse institution et recrute Georges Henri Rivière comme sous-directeur. Rivet détaille le fonctionnement de cette nouvelle direction:

Moi je ferai la science; vous [Rivière]
vous ferez tout ce qui est la traduction
populaire de cette science. Je suis un
homme du peuple. Je veux fonder un
grand musée de culture populaire.
(Laurière 2008: 383)

Dans cette perspective, la muséographie portée par Rivière apparaît comme une nouvelle science soucieuse du «rôle social» du musée en montrant I'unité de I'homme. Outil d'éducation populaire, le musée entend également être un lieu consacré à la recherche (Laurière 2017: 403). Dès le début de son mandat, Rivière souhaite donner des gages de son adhésion au projet scientifique de I'Institut par la production d'un savoir ethnographique sur les objets, conformément aux orientations définies par Mauss 11. Les premières missions, à l'instar de DakarDjibouti, sont organisées directement par la direction du musée avec d'importants moyens logistiques. Rapidement, la plupart des missions font appel, de manière opportuniste, aux étudiants de I'Institut d'ethnologie apprentis chercheurs, souvent bénévoles. Cela permet à une science «de la culture matérielle», en cours de formation et bénéficiant de peu de moyens, de s'adosser à un musée-vitrine crédible doté de collections significatives (Laurière 2017: 409 12).

Maupoil est représentatif de ces jeunes diplômés, formés et envoyés sur le terrain par l'Institut, fortement incités à participer à l'effort de collecte
5. Lettre de Rivet au gouverneur général, Paris, 27 juillet 1933 , CILF.

6. Lettre du gouverneur général à M. Jeanneney, président du Sénat (ami de la famille Maupoil qui soutient la demande d'affectation), Paris, 9 octobre 1933, CILF.

7. Les chefs de canton instaurent une nouvelle forme de pouvoir royal, en réactualisant les symboles. Ils se présentent généralement comme des interlocuteurs privilégiés pour toutes les personnes qui s'intéressent à la culture vodun, missionnaires, ethnologues et autres touristes. À ce sujet, voir: Glélé 1974 : 234-238; Zuccarelli 1973.

8. Lettre de Maupoil à Mauss, Dakar, 26 mars 1937, CILF.

9. Lettre de Mauss à Maupoil 19 avril 1937, Collège de France, 57 CDF 108 20, Mauss.

10. ibid

11. Selon Mauss, l'objet constitue en effet «la preuve du fait social» qu'il convient de classer et d'archiver à des fins d'étude: «Une collection d'objets systématiquement recueillis est done un riche recueil de "pièces à conviction", dont la réunion forme des archives plus révélatrices et plus sûre que les archives écrites [...].», Mauss et al. 1931: 6-7 Mauss et al. 1931: 6-7
[les présentes instructions ont été rédigées d'après les cours professés à l'Institut d'ethnologie].

12. Parmi la centaine de missions réalisées entre 1925 et 1940 , plus des deux tiers le sont par des élèves de l'Institut. 
13. Lettre de Maupoil à Rivet, Paris, 20 juillet 1933, CILF.

14. Lettre de Rivière à Maupoil, Paris, 23 février 1934, MNHN, 2 AM 1 K64e.

15. Lettre de Maupoil à Rivet, Porto-Novo, 3 janvier 1934, MNHN, 2 AM 1 K64e.

16. Lettre de Maupoil à Rivet, Porto-Novo, 24 août 1934, MNHN, 2 AM 1 K64e.

17. Lettre de Rivière à Maupoil, Paris, 13 juin 1934, CILF :

Mlles Paulme et Liffszyc

ont achevé le catalogue

(révisé par Le Hérissé) en juin.

18. Lettre de Maupoil à Mauss Porto-Novo, 22 août 1934, Collège de France, 57 CDF 81-14.

19. Lettre de Rivière à Maupoil, Paris, 14 août 1934, CILF.

20. Lettre de Rivière à Maupoil, Paris, 8 septembre 1934, CILF.

21. Notamment les douze copies de récades à l'emblème des différents rois du Danhomè, réalisées sur une commande

d'Auǵuste Le Hérissé vers 1910 [MQBJC, 71.1931.36.1 à 12]

22. Martine Balard cite le rapport relatif à l'organisation du stand des missions africaines (1998: 115), AMA Rome 3H39: 3

23. Ancien élève de l'école normale de Saint-Louis du Sénégal, instituteur du Sénégal, i

24. Ciarcia 2019: 222 (lettres à Paul Hazoumé, correspondances Aupiais, Rome, SMA-Sank 75 ; Rome, SMA-Sankofa, $75 \mathrm{~s}$ lettre du 12 février 1928 lettres à Paul Hazoumé, Rome SMA-Sankofa, 83 sq.; lettre du 25 juin 1928).

25. Lettre de Rivière à Maupoil, Paris, 14 août 1934, CILF ; Leiris 2003 [1934]:175 (10 décembre, Abomey "Au musée, beaux chassemouches faits de queues de cheval montées sur un crâne humain, avec un manche sous la mâchoire»; Cet objet est mentionné dans le catalogu mentionné dans le cataloǵue
du musée d'Abomey de 1931 du musée d'Abomey de 1931
[en liǵne], disponible sur: http://lalbere.net/siteGG.V4/ Pagesdereferences/Abomey/ Musee/Guide.pdf,

(consulté le 14 décembre 2020).

26. Lettre de Rivière à Maupoil, Paris, 13 octobre 1934, CILF.

27. Lettre Rivet au gouverneur [Reste?], 8 septembre 1934 CILF.

28. Dossier Justin Aho paragraphe donnant des informations sur la création du musée d'Abomey: orédit de 15000 f pour aménagement, par décision du 25 avril 1929 , CILF.

(Voir suite des notes p. 201)
(Jolly 2001). II décide ensuite, nous l'avons vu, d'embrasser la carrière coloniale. Son statut d'administrateur lui apparaît alors comme une formidable opportunité de faire du terrain, tout en étant rémunéré:

\section{J'ai décidé de renoncer, au moins pro- visoirement, au Trocadéro, et de rejoindre mon poste en Afrique comme adminis- trateur. [...] J'espère rester en contact avec le Trocadéro et, de loin, travailler avec vous $\mathbf{1 3}$.}

Dès sa prise de poste à Porto-Novo début 1934, il emploie toute son énergie à la «cause ethnogra-

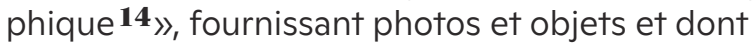
il finance lui-même l'acquisition. II fait également bénéficier le Trocadéro de toutes les informations que sa position lui permet d'obtenir. Dans un premier courrier, quelques jours après son arrivée, il donne à Rivet des noms d'administrateurs à la retraite, collectionneurs «sans enfants» ou initiés, à contacter. Maupoil est, par ailleurs, sollicité pour les différents projets de valorisation du patrimoine dahoméen par l'administration coloniale, en France et sur place. Il participe à la mise en place du musée permanent des Colonies de Vincennes, «commode prétexte pour entrer en contact avec des artisans indigènes ou des collectionneurs du Dahomey $\mathbf{1 5}_{\Perp}$. On lui confie également «la mission de classer, de décrire et de cataloguer les objets rares et souvent très beaux du musée d'Abomey»:

\section{Dès que le classement des objets du musée d'Abomey sera effectué, et les fiches tapées, je vous adresserai pour vos collections une série complète des fiches 16 .}

La proposition faite par Maupoil de collaborer avec le MET tombe à pic, la section du Dahomey devant être installée au moment de sa prise de poste $\mathbf{1 7}$. II supervise ainsi l'envoi «d'une caisse d'objets commandés quelques mois plus tôt par Métraux ou Leiris»,

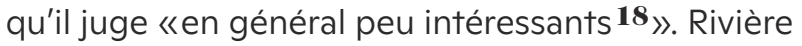
espère que Maupoil pourra l'aider à combler les manques de la section Dahomey en cours d'installation. Outre la vitrine des rois «qui est déjà fort belle $\mathbf{1 9}_{\text {», }}$ il est question de celles consacrées à la religion et aux techniques:

Nous avons eu la bonne fortune de retrouver d'admirables tentures (à application d'étoffes) envoyées en présent parles rois de Dahomeyà l'Empereur Napoléon III. Notez, la série de récades [sceptres royaux] est magnifique [...]. Tout cela joint aux monumentales statues de rois (venant du général Dodds) et aux admirables poteaux rapportés par les missions DakarDjibouti fait un ensemble imposant que vous aurez le plaisir de voir dans quelques jours. Etj'oubliai notre dieu de la guerre, qui préside à la vitrine d'art africain $\mathbf{2 0}$.
Les termes utilisés par Rivière pour décrire la vitrine relèvent de l'appréciation formelle. La plupart des objets présentés proviennent de pillages, de cadeaux «diplomatiques», de commandes 21 ou d'achats à des antiquaires. II n'y a a priori pas eu de démarche de collecte sur le terrain et les objets sont peu documentés. Tout musée de science qu'il prétend être, le MET est surtout là pour susciter émotion, fascination et étonnement auprès du public occidental. Michel Leiris, défenseur d'une démarche proprement ethnologique, confessera lui-même à la fin de sa vie que «les objets d'art sont les plus attrayants» (Dupuis 1999: 518). Cette orientation esthétisante rejoint celle des missionnaires. Le révérend-père Aupiais, responsable des collections dahoméennes pour l'exposition missionnaire du Vatican en 1925, explique que l'objectif est avant tout de donner une bonne image des «indigènes» au grand public:

\section{Il sera convenable que ces curiosités aient un caractère artistique - plutôt qu'un caractère grotesque et odieux - de ma- nière à faire estimer les indigènes $[. . .]^{\mathbf{2 2}}$.}

Selon lui, la contemplation des plus belles pièces, susceptibles de susciter de l'admiration, déboucherait nécessairement sur le respect des producteurs des objets. II tient d'ailleurs le même discours à propos des documents cinématographiques qu'il souhaite réaliser pour le compte d'Albert Kahn et ses Archives de la planète. II demande à cet effet à un de ses élèves dahoméens, Paul Hazoumé $\mathbf{2 3}$, de faire une liste «des plus gracieux usages, des plus belles cérémonies

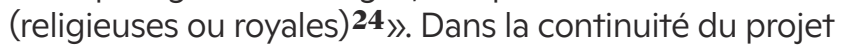
missionnaire de promotion des sociétés africaines, Rivière demande à Maupoil de trouverun certain nombre de «beaux objets». II souhaite notamment obtenir «un chasse-mouches» «parmi ceux qui sont conservés au musée d'Abomey, évoqués par Leiris $\mathbf{2 5}_{\Downarrow}$. Il ambitionne également de pouvoir présenter «un très beau spécimen (je parle au point de vue de l'art) de plateau divinatoire, travail ancien du genre de ceux dont Frobenius a enrichi plusieurs musées allemands» et «enrage de voir qu'une pièce de ce genre [leur] manque $\mathbf{2 6}_{\gg \text {. }}$

Le musée d'Abomey, fraîchement inauguré en 1930, apparaît dans ce contexte comme un moyen d'obtenir des objets pour le MET, d'autant que certains sont réputés exister «en double». Rivet écrit au gouverneur François Reste, son fondateur, pour faciliter les dons, avec la contrepartie d'en assurer la meilleure publicité possible $\mathbf{2 7}$. La création d'un musée historique dans l'une des cases du palais des rois d'Abomey, regroupant «les meilleurs spécimens de l'art dahoméens en même temps que les objets ayant appartenu aux anciens rois $\mathbf{2 8}_{\gg,}$, s'inscrit dans une démarche de sauvegarde du patrimoine local, déclinaison du courant régionaliste français au cœur des colonies (Thiesse 1996: 2) 29. La direction en est confiée à Justin Aho Glélé, neveu de Béhanzin et chef de canton. Comme l'a expliqué Maurice Ahanhanzo Glélé, ces chefs de canton ont 
L'expérience de Bernard Maupoil au Dahomey : entre science et engagéement, un laboratoire pour l'ethnologie en milieu colonial Valérie Perlès



Plateau de divination qui appartenait au bokonon royal Gèdègbé, bois, bleu de lessive, kaolin, 42,3 × 35,1 × 1,7 cm, $1126 \mathrm{~g}, \mathrm{n}^{\circ}$ inv. 71.1938 .17 .3 (c) musée du quai Branly-Jacques Chirac. 


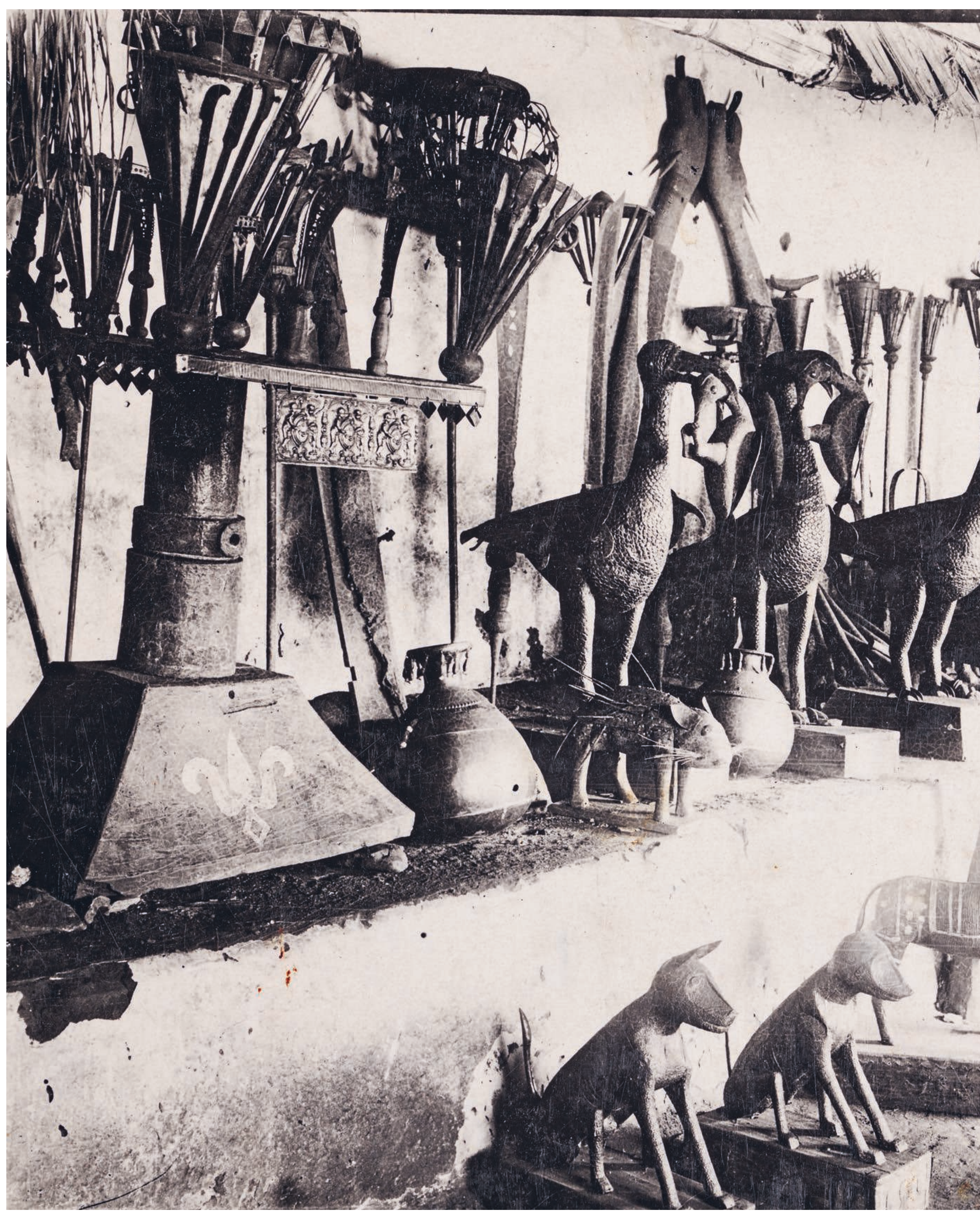


Études et essais

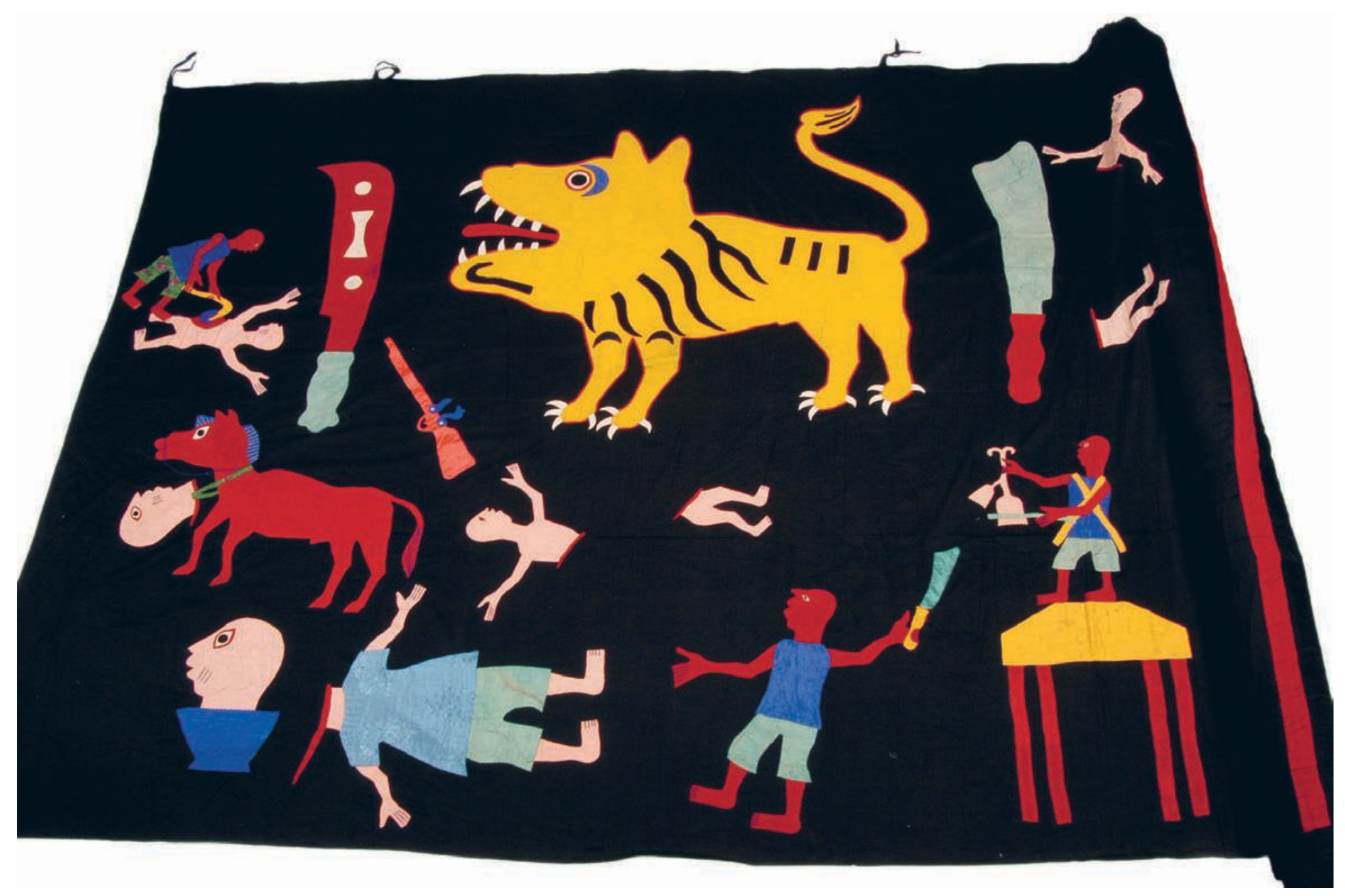

Tenture comportant les symboles des principaux rois du Dahomey, coton armure toile, application, $1840 \times 201 \times 1 \mathrm{~cm}, 6979 \mathrm{~g}, \mathrm{n}^{\circ} \mathrm{inv} .71 .1936 .21 .28$ (c) musée du quai Branly-Jacques Chirac. 
eu tendance à perpétuer la mémoire des rois en la détournant à leur profit30. Au sujet de l'ascension sociale de Justin Aho, Glélé cite le rapport du commandant de cercle d'Abomey en 1949, qui critique sa nomination à la fonction de «conservateur», le promouvant ainsi «gardien des trônes, des bijoux, des assens [autels portatifs représentant les ancêtres], des fétiches royaux» (Glélé 1974: 237-238, cité par Ciarcia 2019: 231). On mesure alors pleinement l'importance de ces regalia au cœur de la stratégie de légitimation d'Aho et la réticence à s'en dessaisir. Elles sont d'ailleurs immortalisées par la caméra de Gadmer, I'opérateur d'Albert Kahn qui a collaboré avec Aupiais en 1931 pour les Archives de la planète $\mathbf{3 1}$.

Maupoil conseille donc à Rivet de tenter la voie diplomatique, flattant l'ego de «ce chef très influent et conservateur du musée» lors de sa prochaine venue à Paris. Si les négociations venaient à aboutir, des copies devraient être faites pour remplacer les objets donnés au MET32. Maupoil n'arrive finalement pas à avoir les objets attendus par Rivière, «même ceux en double». Cette situation, certes en partie liée au climat de compétition qui s'instaure «entre les musées installés dans les colonies et l'institution métropoli-

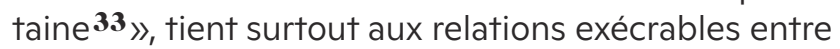
le chef dahoméen et le jeune ethnologue. Ce dernier souligne d'ailleurs dans sa thèse la gestion malhonnête du «conservateur», l'accusant explicitement de vol:

Il est regrettable qu'un catalogue méthodique des objets contenus dans ce musée [d'Abomey] n'ait pas encore été dressé. Il est de notoriété publique que des pièces intéressantes ont disparu. (Maupoil 1943: 183)

Face au peu de résultats obtenus par Maupoil, l'équipe du MET s'impatiente. Denise Paulme est très lucide sur les objectifs du musée à court terme, qu'elle juge plus idéologiques que scientifiques. Elle-même chargée de la rédaction du catalogue et de la mise en place de la section Dahomey, elle reconnaît que cette dernière est «vaseuse». Avec une très grande franchise, elle explique à Maupoil que le musée a davantage besoin de collections que d'ethnologues, que lui-même souffre d'une réputation de dilettante auprès de «Marcel [Griaule?], Paul [Rivet?] et la bande», en qui ils voient un «garçon brillant et peu sérieux, qui vous parle trop de ses projets, entreprend beaucoup et ne produit rien». Elle lui conseille de faire passer la collecte en priorité:

Envoyez un travail quelconque, envoyez des objets, n'importe quoi, mais faites vite. Faites une séparation nette entre votre travail pour vous et ce que vous enverrez à Paris, mais ayez l'air de faire quelque chose. Ne demandez pas aux gens ce qu'ils veulent, mais assommez-les sous le poids d'un costume de féticheur (de bazar), de poteries ramassées sur le marché, etc. et écrivez moins $\mathbf{3 4}$.

Pour donner satisfaction au Trocadéro, Maupoil entreprend donc de faire fabriquer des objets, parfois sur le modèle de ceux conservés au musée d'Abomey. Les copies sont alors réalisées de manière clandestine, sans qu'Aho soit mis au courant. II confie la coordination des commandes au «fils préféré» de son principal informateur, Ernest Gèdègbé, qui deviendra par la suite un fidèle collaborateur. En février 1935, il commande «une tenture d'une 20taine de $\mathrm{m}$. de long sur $3 \mathrm{~m}$. de haut, étoffe noire à motifs de couleur rapportés, et constituant I'histoire du Dahomey,

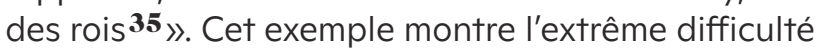
de mener à bien ce type d'entreprise, du fait notamment de la multiplication des intervenants et de leur manque de fiabilité. Ernest Gèdègbé demande de l'argent à plusieurs reprises, dépassant largement l'enveloppe initialement prévue $\mathbf{3 6}^{\mathbf{3 6}}$. L'artisan chargé du chantier, Dossou Nanmankpon, I'accuse même d'en avoir détourné une partie et d'avoir trahi la confiance de Maupoil 37. La copie du «pagne du musée royal», finalement achevée, est réquisitionnée par Aho:

\begin{abstract}
Un chef de canton, Justin Aho, véritable canaille, [...] s'amuse en ce momentà me voler. J'ai commandé une grande tenture, les blasons du roi. II m’a volé la première pièce et met l'embargo sur la seconde. J'ai dépensé 900 fcs pour cela. J'ai fait d'autres commandes égalementstoppées sur son ordre $\mathbf{3 8}$.
\end{abstract}

À la fin de la même année, Maupoil commande également «une scène de la vie dahoméenne, montable sur planche, à 32 figurines de cuivre ${ }^{\mathbf{3 9}} \gg$, saynète représentant les coutumes d'Ato, copie d'un objet présent au musée d'Abomey. Comme pour les tentures, Ernest est chargé de la coordination de la fabrication, confiée à des charpentiers, et de la finalisation de la décoration. II indique un important retard et se plaint de l'ampleur et de la complexité de la commande, ainsi que de son coût:

\section{Presque tous les objets en cuivre sont achevés. [...] Je voulais vous les apporter mais les décorateurs sont toujours em- bêtés par le chef qui leur avait donné une liberté superficielle [pour se déplacer au musée?]. De ce coup, ma présence devint nécessaire pour les aider. Comme il était question qu'ils iraient copier le modèle au musée c'était pour vous plaire. Je me débrouille pour achever la décoration $\mathbf{4 0}$.}

Il est probable que, là aussi, Aho ne leur facilite pas la tâche, étant lui-même à l'initiative d'une commande semblable auprès de la corporation des forgerons. À ce stade, nous n'avons pas bien cerné l'intérêt pour Aho de faire réaliser des copies des objets conservés au musée que lui-même administre. Les recherches devront être approfondies sur ce point.
29. Le mouvement régionaliste, né sous la III République, fait du sentiment d'appartenance locale une propédeutique indispensable au sentiment d'appartenance nationale. Selon cette dialectique, l'avenir du Dahomey s'envisage d'abord, pour les missionnaires comme pour les acteurs de l'action coloniale, sur la base du respect des traditions existantes.

30. Ahanhanzo 1974: 234-236, cité par Ciarcia 2019: 230-231: «En nommant des princes de sang comme chefs, l'administration française reconstitue des petites monarchies, en parfaite contradiction avec la tradition royale dahoméenne qui écartait du pouvoir et de l'administration tous les princes. »

31. MDAK, AI13575, cérémonies d'hommages aux ancêtres royaux chez Justin Aho Lieux Oungbégamè, environs d'Abomey, 16-18février 1930.

32. Lettre de Maupoil à Rivet, Porto-Novo, 19 novembre 1934, mention «personnelle», MNHN, 2 AM 1 K64e.

33. Lettre de Maupoil à Rivet, Porto-Novo, 9 juillet 1934 MNHN, 2 AM 1 K64e.

34. Lettre de Paulme à Maupoil, Paris, 5 novembre 1934, CILF.

35. Maupoil à Rivet, Athiémé, 17 février 35, MNHN, 2 AM 1 K64e.

36. Lettres d'Ernest Gèdègbé à Maupoil, Abomey, 19 septembre et 27 octobre 1935 (notamment pour acheter des pagnes de différentes couleurs à la maison Ghon-Holte), CILF

37. Lettre de Dossou Nanmankpon à Maupoil, Abomey, 5 janvier 1936, CILF

38. Lettre de Maupoil à Mauss, Cotonou, 22 novembre 1935, 57 CDF 81-14.

39. Lettre de Rivet à Mauss, Paris, 14 janvier 36 : engagement à dédouaner à la gare d'arrivée à Paris une caisse contenant des objets destinés à figurer dans les collections, CILF.

40. Lettre d'Ernest Guédégbé à Maupoil, Abomey, 8 décembre 1935, CILF. 
41. Lettre de Maupoil à Rivet, Athiémé, 17 février 35, MNHN 2 AM 1 K64e.

42. Lettre de Rivière à Maupoil, Paris, 16 mars 1935, MNHN, 2 AM 1 K64e.

43. Lettre de Maupoil à Rivière, Porto-Novo, 22 avril 1935 MNHN, 2 AM 1 K64e.

44. Probablement MQBJC, inv.71.1936.21.66. La tunique a apparemment été cousue au sein de l'atelier de la famille Yémadjé : voir: Beaujean-

Baltzer (dir.) 2009: 52.

45. Gaëlle Beaujean, communication personnelle, 22 avril 2020.

46. Lettre de Maupoil à Rivet, Cotonou, 13 janvier 1936 MNHN, 2 AM 1 K64e.
Le recours à la copie permet donc de contourner une négociation souvent difficile pour obtenir un objet encore inscrit dans une logique d'usage, déjà patrimonialisé ou dont la possession procure un prestige important. La question de «l'original» ne semble pas décisive, puisque ces copies ont, pour la plupart, la même valeur matérielle que des objets ayant servi ou servant encore. Comme l'a montré Gaëlle Beaujean, un grand nombre d'objets royaux conservés au musée d'Abomey ont été recréés à l'identique à la demande du roi Agoli-Agbo - frère et successeur de Béhanzin - pour remplacer ceux qui avaient été volés ou détruits pendant la guerre (notamment les trônes utilisés lors des Coutumes annuelles) (Beaujean 2015: 73 et 111). Gèdègbé, devin des rois, grand sage ayant accepté de transmettre ses connaissances à Maupoil, fait également fabriquer une copie du «calendrier de Fa destiné aux consultations du roi» à son intention:

Cetobjeta perdu beaucoup de son prestige depuis la chute de la monarchie. Il estencore profondément respecté dans la famille du dernier grand bokono de la cour, Gèdègbé. [...] Gèdègbé, qui nous l'offrit, le fit exécuter à l'imitation du sien. (Maupoil 1943: 217, n.2)

II n'est, en effet, pas concevable que Gèdègbé donne autre chose qu'une copie à l'ethnologue français. Un objet en usage dans un cadre religieux, nécessairement «chargé», peut s'avérer dangereux pour un profane. En revanche, un autre, nouvellement fabriqué, peut tout à fait être collecté pour un musée (Amouro 2009: 42-43).

Parfois, au-delà de l'objet lui-même et de ce qu'il représente, c'est le savoir-faire ici employé que I'on cherche à sauvegarder. Maupoil propose par exemple à Rivière de faire confectionner les costumes des grands fonctionnaires des rois par un artisan qui, eut égard à son grand âge, risque de mourir «en emportant son secret»:

L'artiste vit encore, et pourrait faire une reproduction parfaite, soit pour vous, soit pour les familles qui donneraient en échange les anciens. Je vous préviens que ce serait cher et il faudrait une moyenne de $1000 \mathrm{fcs}$ par costume, ministre, chef de guerre. II y a de l'argent et de l'or. L'ensemble est somptueux et va tomber sous peu dans un parfait oubli41

Rivière est intéressé mais trouve le prix «mathématiquement prohibitif» et propose donc d'utiliser des matériaux moins onéreux pour la copie:

Ne pourrait-on exécuter à prix bien moindre sans or, en argent doré? À quel prix nous mènerait un costume de chef de guerre $\mathbf{4 2}$ ?
Maupoil s'y oppose car, selon lui, au-delà de son apparence ce sont la qualité intrinsèque du vêtement et les matériaux utilisés qui font sens. Remplacer I'or par une imitation serait un grave contresens:

De grands chefs comme le roi de la mort ne se laissent pas servir par des gens en guenilles. [...] Ne pas oublier qu'il ya des bracelets d'argent incrustés d'or, et que la camelotte est un produit de civilisations évoluées, du genre de la nôtre $\mathbf{4 3}$.

Maupoil ramène trois tuniques de guerre et celle

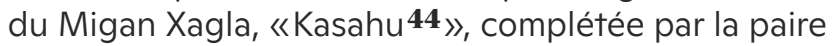
de bracelets «Ma-wu-alo» (tu-ne-plieras pas-le- bras), un jonc noué en forme de serpent et une paire de bracelets de cheville, le tout en argent. II n'y a manifestement pas d'or ni sur la tunique, ni sur les parures. Lorsque l'on considère les nombreuses copies réalisées à la demande de Maupoil, d'Aho voire de Gèdègbé, et les échanges dans la correspondance avec Rivière, on peut se demander si la panoplie du Migan Xagla n'est pas, elle aussi, une copie. Comme l'observe néanmoins Gaëlle Beaujean, la présence d'une mandibule humaine cousue à l'intérieur, qui renvoie à la délicate question des sacrifices humains et de la manipulation des cadavres, permet d'en faire douter $\mathbf{4 5}$. L'enquête sur l'origine de ces objets royaux et la manière dont Maupoil les a obtenus mériterait en tout état de cause d'être poursuivie.

Maupoil finance très largement ces acquisitions, témoins de l'obligation des jeunes chercheurs de I'Institut à collaborer avec l'institution muséale, même lorsque la collecte s'éloigne de leurs sujets de recherche et répond à une commande parfois en décalage avec les réalités rencontrées:

J'ai pris à mon compte toutes les dépenses occasionnées par les objets que vous avez reçus. Tout est payé. Les dépenses s'élèvent à $7000 \mathrm{fcs}$. Vous verrez dans quelles mesures vos disponibilités vous permettent de me désintéresser: en tout cas, je tiens à vous écrire que je ne compte sur rien. J'ai agi par dévouement pour l'œuvre que vous poursuivez, etje pense que vous ne douterez pas de ce dévouement $\mathbf{4 6}$.

À côté des objets d'apparat, difficiles à obtenir à cause des tensions avec le musée d'Abomey et son conservateur, Maupoil rapporte une collection importante d'objets liés au Fa et à la pratique des rituels vodun, davantage en lien avec les recherches de terrain pour sa thèse consacrée à la divination. Comme nous allons le voir, contrairement aux «beaux» objets demandés par Rivière, la question de l'usage est ici déterminante, remettant parfois en cause la dimension matérielle de la collecte. 
L'expérience de Bernard Maupoil au Dahomey : entre science et engáagement, un laboratoire pour l'ethnologie en milieu colonial Valérie Perlès

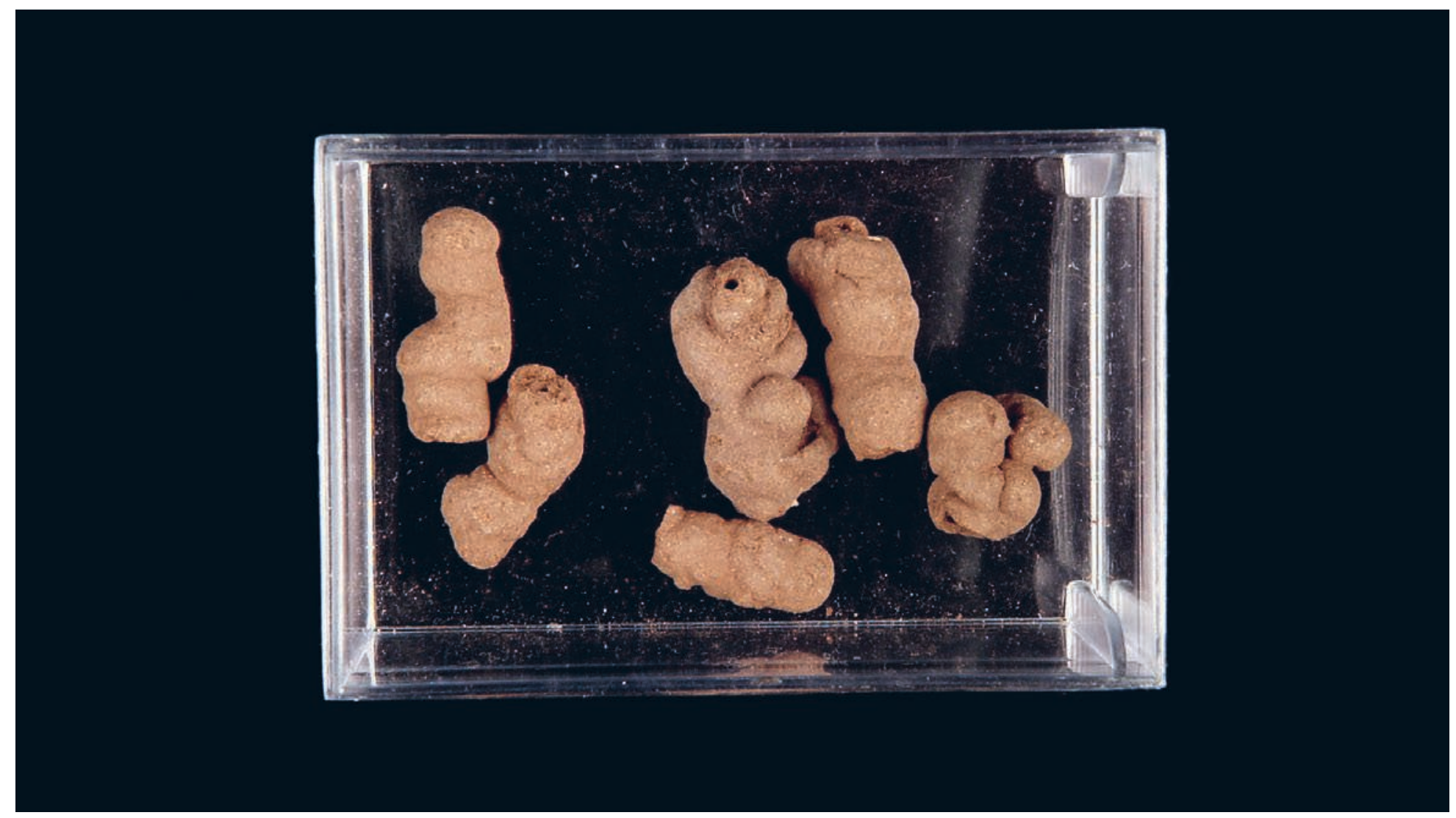

Noix de palmes collectées par Bernard Maupoil, $n^{\circ}$ inv. 71.1938.17.28 @ musée du quai Branly-Jacques Chirac.

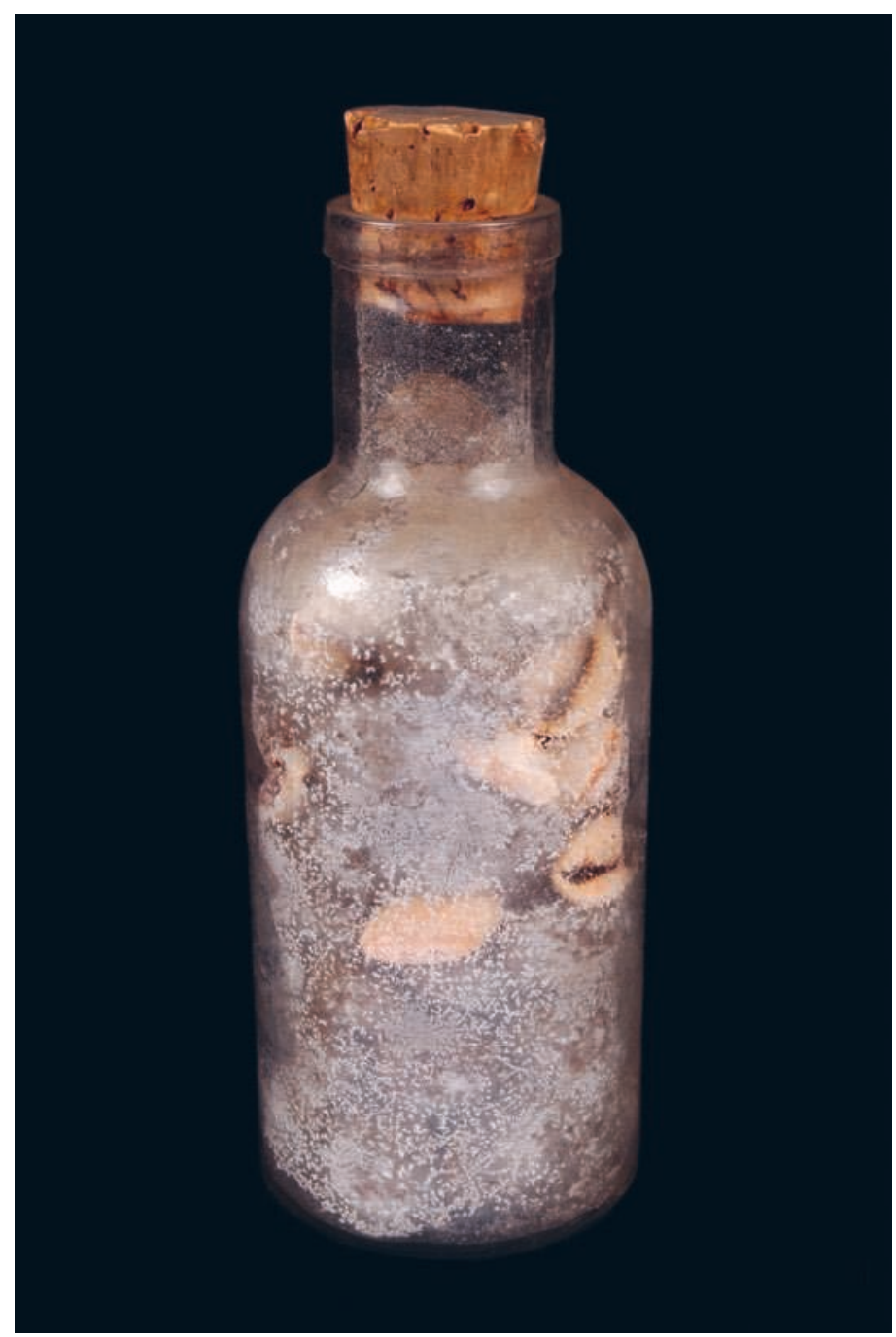

Échantillons de terre de barre, collectés par Bernard Maupoil, n inv. 71.1938.17.27.1-6 @ musée du quai Branly-Jacques Chirac. 
Études et essais

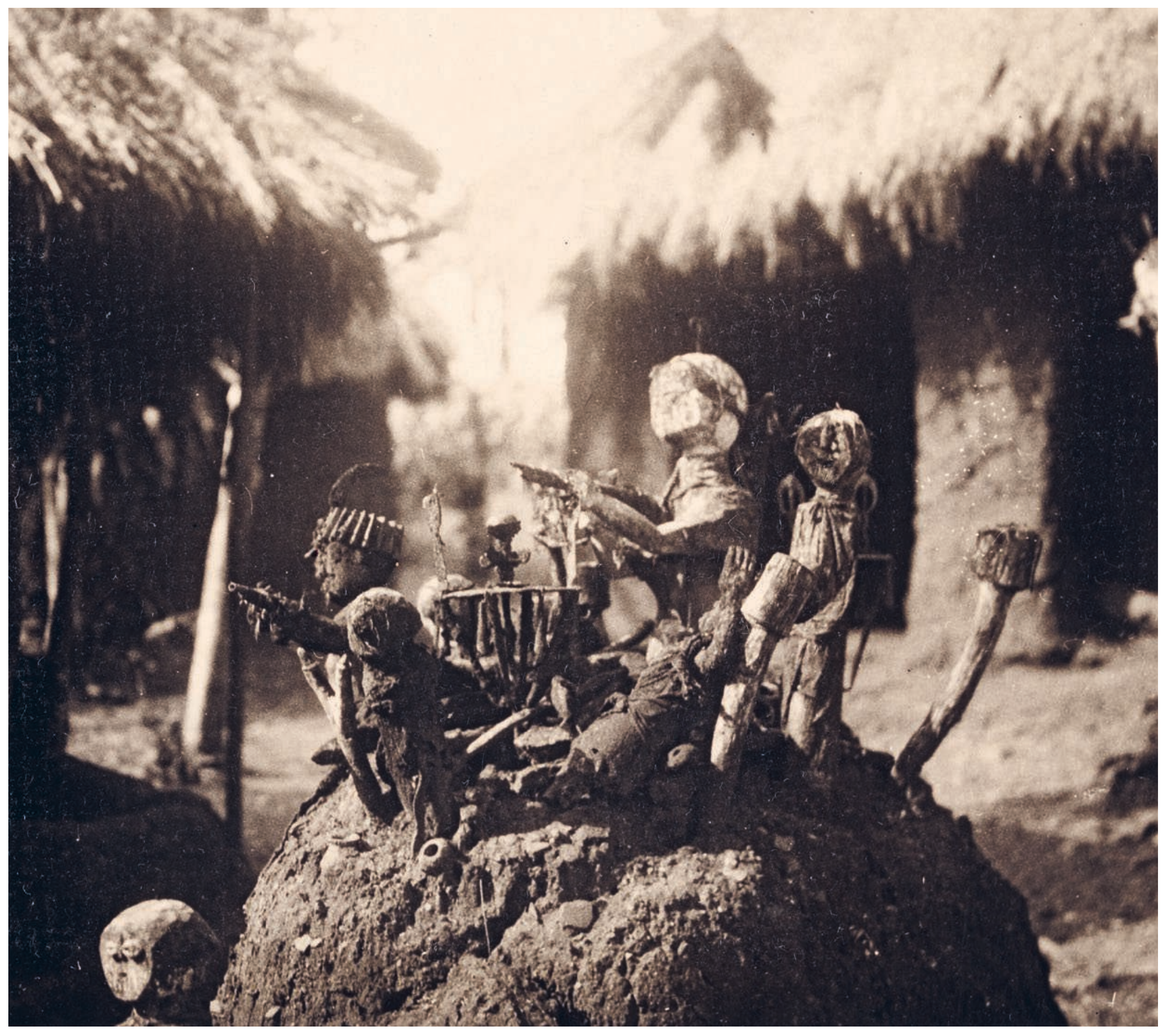

Bernard Maupoil, Couvent du Tonnerre: autel du Tonnerre, cf. D.39-788 et 789, Bopa, 1934-1936, tirage sur papier baryté monté sur carton, 22,5 × 29,5 cm, $n^{\circ}$ inv. PP0036487 @ musée du quai Branly-Jacques Chirac. 


\section{L'OBJET PALIMPSESTE: CRISTALLISATION D'UNE DÉMARCHE SCIENTIFIQUE}

Malgré la structuration de la jeune discipline ethnologique autour de l'étude de la culture matérielle, les objets choisis pour rejoindre les collections du MET, nous l'avons vu, ne sont pas forcément documentés et la collecte apparaît souvent comme une démarche nécessaire mais superficielle. La mission Dakar-Djibouti, érigée en modèle, repose sur une pratique extensive, rapide et efficace, qui vise à ramener des séries complètes d'objets en rapport avec des phénomènes observés. Griaule, lui, rejette l'idéal d'une ethnographie intensive qui, sur le modèle anglo-saxon, prône le séjour prolongé sur le terrain (Hirsch 2017: 370). L'urgence de la collecte est d'ailleurs dénoncée par Leiris dans L'Afrique Fantôme:

Les méthodes de collecte des objets sont, neuf fois sur dix, des méthodes d'achat forcé, pour ne pas dire de réquisition. [...] on pille des Nègres, sous prétexte d'apprendre aux gens à les connaître et les aimer, c'est-à-dire, en fin de compte, à former d'autres ethnographes qui iront eux aussi les «aimer» et les piller.

(Leiris 1996: 204, 19 septembre 1931, lettre à Zette, citée par Leborǵne 2017)

Ce paradoxe entre une démarche sincère de connaissance et l'acceptation d'une forme de violence pour arriver à ses fins, est dénoncé par de nombreux ethnologues, qui finissent cependant par se plier à ces méthodes. Pour reprendre les termes de Paulme, une mission qui reviendrait les «caisses vides $\mathbf{4 7}_{\text {》 }}$ est en effet impensable. Le séjour au Dahomey a d'ailleurs fait l'objet d'un investissement scientifique très relatif de la part de la mission Dakar-Djibouti, les trois cent quatre-vingt-dix objets collectés à la hâte ayant été peu documentés. À Porto-Novo, un «stock énorme de poteries et de ferrailles rituelles» est acheté sur le marché; un jeune fonctionnaire «habitué du Trocadéro» y fait un don important, incluant une collection $d^{\prime}$ '«amulettes» et d'«objets magiques» provenant de Bopa, saisie par la police:

Nous nous transformons en entreprise de déménagement, caril nous fait don de plus de 50 objets, que nous emportons surl'heure avec un cynisme de businessmen ou d'huissiers (12 décembre, Porto-Novo). (Leiris 2003 [1934]: 178).

Le matériel liturgique croisé en chemin semble plutôt inspirer du mépris, voire du dégoût:

Retour à la résidence après un passage devant un legba d'au moins trois mètres de haut, gigantesque monceau d'immondices, couleur fumier mêlé de cet étrange jaune d'œuf que devient I'huile de palme en se coagulant. (11 décembre, Ouidah) (ibid.: 177)
Ce manque d'intérêt est certainement lié au type d'objets présents dans les rituels vodun, qui sont rarement «beaux» comme en témoigne Maupoil dans sa thèse:

La tendance à anthropomorphiser est peu développée au Dahomey. Une motte de terre, une poterie spéciale, une couleur, des espèces végétales ou animales symbolisent des vodun mieux qu'une statue, et il ne s'agit nullement là d'incapacité artistique. Fa, de l'avis des meilleurs devins, n'est pas susceptible de représentation, anthropomorphes ou autres. On peuttout au plus le comparer à diverses entités, - le plus souvent à la vérité -, ou à certains objets - ceux qui forment la base de son culte: noix de palme, palmier spécial -, ou encore à quelques animaux qui symbolisent la puissance [...].

(Maupoil 1943: 18)

Lorsque Rivière demande à Maupoil de rapporter «un très beau spécimen (je parle au point de vue de l'art) de plateau divinatoire» pour les salles du MET, il fait donc un contresens au nom de motivations purement esthétiques $\mathbf{4 8}$. En effet, au sujet du «plateau à 16 têtes de Gèdègbé», Maupoil indique que «de bons informateurs nient que les sculptures marginales des Fate aient un symbolisme quelconque, et accusent de charlatanisme ceux qui disent le contraire» (ibid. 192). La référence à la «souillure», clairement mentionnée à plusieurs reprises dans les notes de Leiris au sujet du matériel liturgique, est confirmée par Maupoil qui l'explicite et en révise la dimension péjorative:

À la surface de certains d'entre eux [objets consacrés à Fa], I'huile de palme, l'alcool, les petites plumes, le duvet et le sang des volailles sacrifiées, une poule blanche et diverses «nourritures» forment un enduit qui est loin d'être considéré comme une souillure, et porte le nom de cekpe.

(ibid: : 178)

Comme l'indique Nanette Jacomijn Snoep, commissaire de la remarquable exposition «Bazar des dieux ou figures de l'informe», présentée au musée du quai Branly en 2009, I'usage de matières coulantes (sang, œuf, huile, alcool), qui se transforment et transforment l'objet avec le temps, est fondamental pour faire fonctionner le matériel de divination. Ce n'est pas tant sa forme qui détermine sa fonction que ce qu'il cache, ce qu'il contient et qui est souvent invisible (Snoep 2009: 31-32). L'objet collecté par Maupoil n'a donc de sens qu'à travers la prise en compte de sa manipulation au sein du rituel, au-delà de sa qualité formelle. Dans un courrier qu'il envoie au MET, il se préoccupe de la préservation des traces d'usage et probablement du cekpe, croûte sacrificielle formée par la stratification des sacrifices successifs:
47. Lettre de Paulme à Maupoil, Paris, 5 novembre 1934, CILF

48. Lettre de Rivière à Maupoil, Paris, 13 octobre 1934, CILF. 
49. Lettre Maupoil à Mlle?, Cotonou, 5 novembre 1935 ; note manuscrite, 2 décembre 1935 de Michel Leiris : «prière de donner numéro à la collection et peut-être écrire à Maupoil », CILF.

50. Lettre de Leiris à Maupoil, Paris, 2 décembre 1935, CILF.

51. Lettre de Leiris à Maupoil, 7 décembre [1935], GILF.

52. Probablement MQBJC, inv.71.1935.116.83.

53. Lettre de Maupoil à Mlle [illisible], Cotonou 16 novembre 1935 , CILF.

54. Lettre de Maupoil à Mauss, Athiémé, 30 mars 1935, 57 CDF 81 14, probablement MQBJC, inv. 71.1936 .21 .54 et 71.1936 .21 .55 .
Chacun des objets parvenus récemment en France était une divinité, possédait une case, et faisait l'objet d'un culte. II y aurait intérêt à ne pas trop nettoyer, désinfecter, etc. $\mathbf{4 9}$

Leiris est sensible à l'argument selon lequel la conservation d'un objet ethnologique implique, dans la mesure du possible, de préserver les indices liés à son contexte d'utilisation, démarche encore inédite au sein du musée:

Votre collection est des plus intéressantes. Soyez certain que je veillerai à ce qu'on ne lui fasse rien perdre de son authenticité, sous prétexte de la nettoyer $\mathbf{5 0}$.

La surface de la paire de marteaux de divination Ionflin, qui aurait appartenu au bokonon Tosujede, comporte encore aujourd'hui des plumes et du sang coagulé, confirmant la mise en œuvre de ce principe. Un autre courrier fait référence à une «recette» pour la conservation donnée par Maupoil:

Ce sera peut-être la première fois que, dans notre laboratoire, sera mis en œuvre un procédé indigène de conservation. Je souhaiterais presque voir cela s'étendre à l'ensemble de l'ethnographie $\mathbf{5 1}$ !

Nous n'avons pour l'instant pas retrouvé le contenu de cette «recette», mais son existence atteste de la prise en compte, au sein du musée, de la dimension immatérielle du fait rituel par la reproduction de gestes et pratiques inhérents à l'utilisation et à l'entretien de l'objet.

En proposant des objets encore en usage, Maupoil se conforme aux orientations «ethnographiques» données par les «instructions sommaires...», selon lesquelles le collecteur ne doit pas tenir compte de «critères tels que la beauté, la rareté, l'ancienneté, le pittoresque ou la pureté de style». Ce parti pris anti-esthétique n'a de sens que si l'objet reste «vivant», relié à son contexte d'utilisation:

C'est en entourant l'objet d'une masse
de renseignements, techniques ou autres,
et de toute une documentation (photos,
dessins, observations) qu'on parviendra
à éviter qu'une fois dans le musée, il se
transforme en objet mort, abstrait de
son milieu et incapable de servir de base
à la moindre reconstitution.
(Mauss et al. 1931: 10)

Maupoil détaille ainsi dans sa thèse le contexte d'utilisation de certains objets. Par exemple, au sujet d'une «chaîne divinatoire» métallique, il explique que celle-ci est atypique, voire aberrante au regard de son usage rituel avéré. II s'agit d'un objet syncrétique, peut-être un «souvenir historique», dont les matériaux sont incompatibles avec sa fonction magique:
Le musée de l'Homme possède un agumaga [chapelet réalisé de préférence avec des éléments en lien avec l'eau] de cuivre. [...] Un forgeron de Porto-Novo le fondit à l'époque de la conquête du Dahomey par les Français en utilisant des étuis de cartouches. Le vieux devin Yoruba qui nous le vendit fit une excellente affaire, nous dit-il, car «un agumaga de cuivre, si joli soit-il, ne saurait dire la vérité: il vient du feu $\mathbf{5 2}$ 》.

(Maupoil 1943: 199)

Soucieux de documenter l'utilisation et la production des objets, Maupoil envoie d'ailleurs au musée un certain nombre «d'ingrédients» employés dans la fabrication des amulettes ou durant le rituel: poudres, poivre, noix de palme, terre de barre, perles, etc.

Maupoil insiste auprès de Rivière sur le fait que les objets liés à l'accomplissement des rituels sont «difficiles à acquérir». Une telle collecte suppose I'instauration d'une relation de confiance avec les personnes susceptibles d'apporter ou de commenter les objets. Maupoil s'inquiète de la bonne réception d'une caisse envoyée au MET courant 1935:

Comme je n'aime pas ramasser des objets avec un revolver à la main, il a fallu beaucoup parlementer, et prendre dans la société noire des points d'appui solides, et des avocats $\mathbf{5 3}$.

II met en exergue l'impossibilité de faire de la «vraie» ethnologie dans un contexte de rapport de force déséquilibré. L'accès aux objets à forte valeur rituelle suppose une excellente connaissance du terrain et de ses acteurs. Maupoil décrit à Mauss, de manière un peu théâtrale, comment il a obtenu les «sabres à décapiter » inscrits sur la liste des envois au MET et sur l'origine desquels il faudrait creuser davantage:

Je commande des objets d'Abomey, et on m'a apporté récemment trois couteaux à décapiter des rois: je les destine naturellementau Trocadéro; ce sont des pièces assez peu intéressantes artistiquement, mais d'une telle valeur magique que celui qui me les remit tremblait de tous ses membres. J'essaie d'éviter la peine de mort à mon informateur, etj'espère qu'on ne lui fera aucune histoire à mon départ54.

Malgré la facilité avec laquelle Maupoil obtient des éléments généralement soustraits à la connaissance des Blancs (peut-être grâce à sa relation particulière avec Gèdègbé sur laquelle nous reviendrons), l'ethnologue dans sa thèse s'inquiète de la déontologie. Selon lui, tout ne doit pas être montré, surtout lorsque la divulgation d'informations réputées secrètes représente un danger pour l'intégrité du système étudié. Un confrère administrateur de Maupoil, qui a observé le vodun avant d'être muté, lui indique que ses 


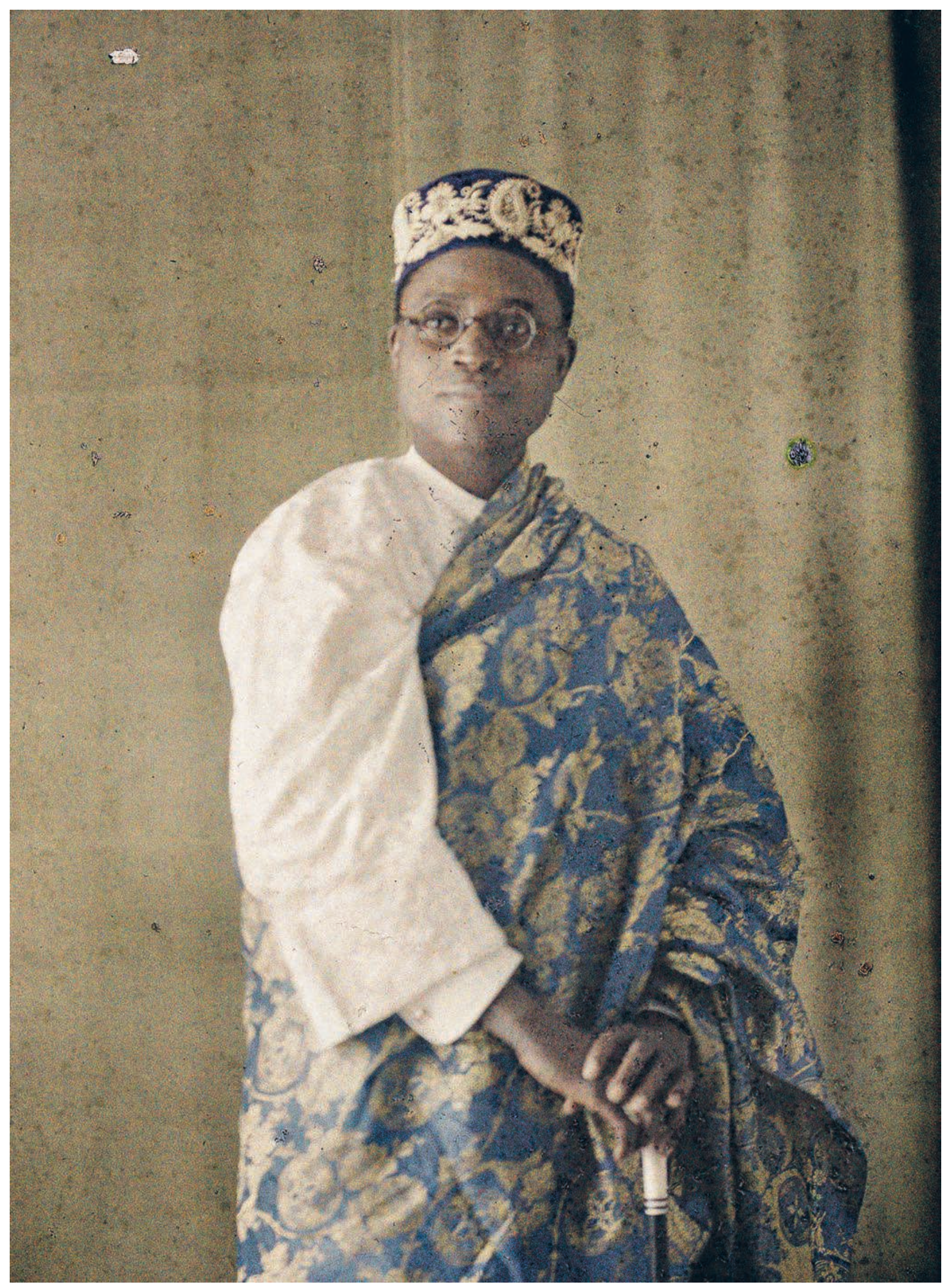

Roger Dumas, Monsieur Paul Hazoumé, réalisé dans le studio d'Albert Kahn à Boulogne le 24 septembre 1931 , autochrome $12 \times 9 \mathrm{~cm}, \mathrm{n}^{\circ}$ inv. A66026. Collection Archives de la Planète - Musée Albert-Kahn/Département des Hauts-de-Seine, collections.albert-kahn.hauts-de-seine.fr 


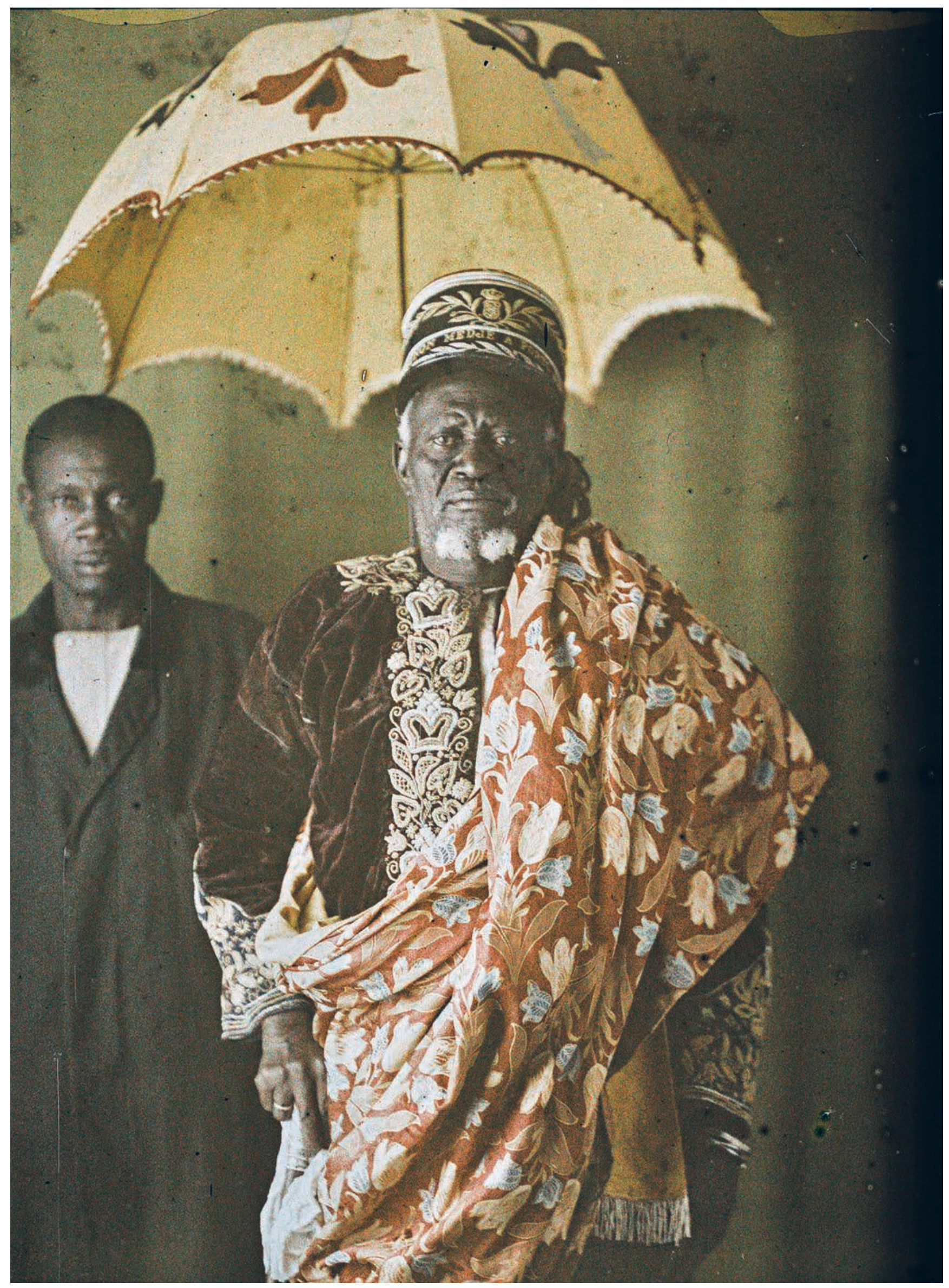

Georges Chevalier, Le Roi Zounon Mèdje, réalisé dans le studio d'Albert Kahn à Boulogne le 17 juillet 1931, autochrome $12 \times 9 \mathrm{~cm}, \mathrm{n}^{\circ}$ inv. A 65683 . Collection Archives de la Planète - Musée Albert-Kahn/Département des Hauts-de-Seine, collections.albert-kahn.hauts-de-seine.fr 
L'expérience de Bernard Maupoil au Dahomey : entre science et engagement, un laboratoire pour l'ethnologie en milieu colonial Valérie Perlès

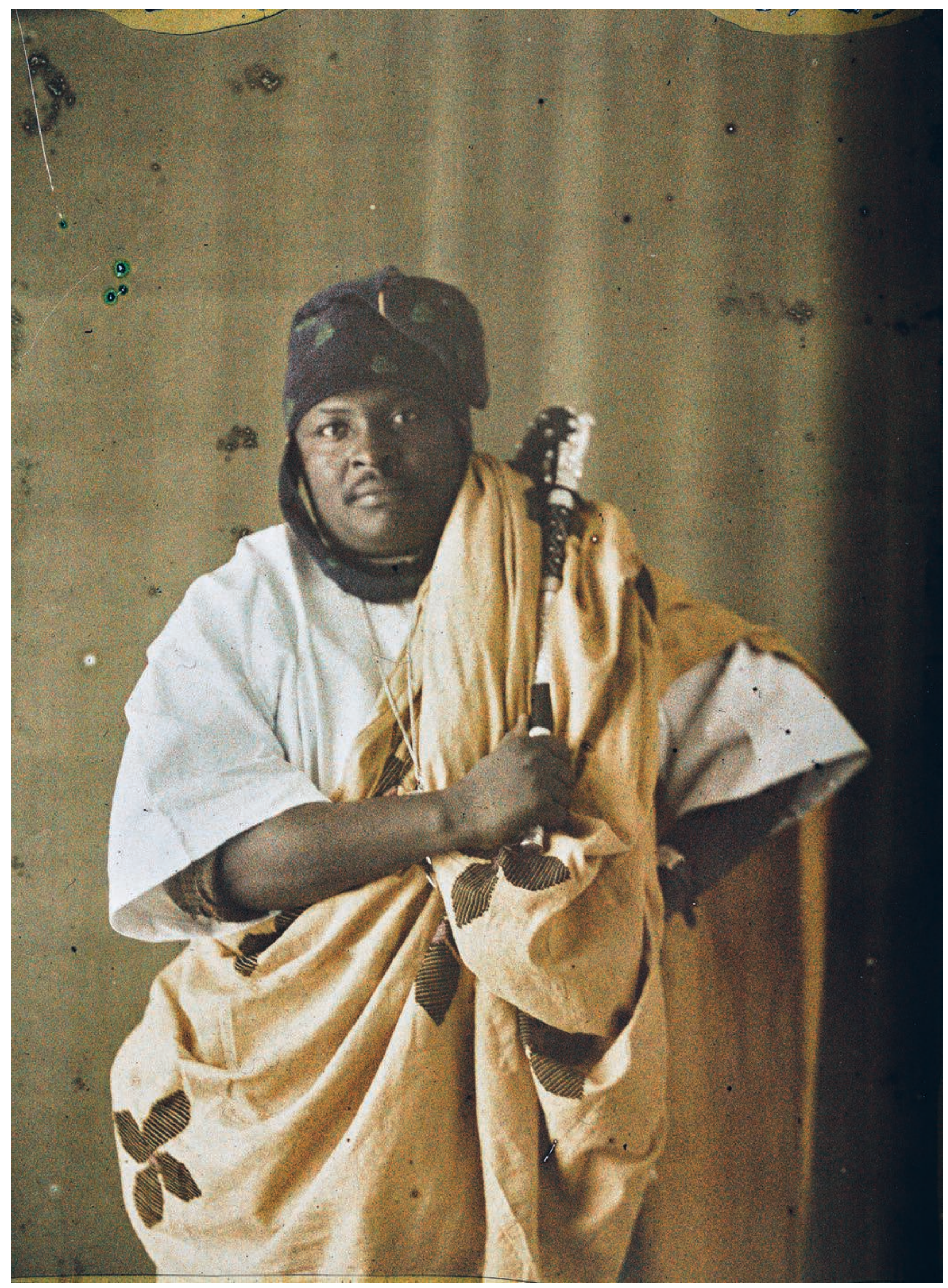

Georges Chevalier, Le Chef Justin Aho, réalisé dans le studio d'Albert Kahn à Boulogne le 11 juillet1931, A65680, autochrome $12 \times 9 \mathrm{~cm}, \mathrm{n}^{\circ}$ inv. A 65680 . Collection Archives de la Planète - Musée Albert-Kahn/Département des Hauts-de-Seine, collections.albert-kahn.hauts-de-seine.fr 
55. Lettre de [illisible, administrateur] à Maupoil, Séméré, 6 octobre 1934, CILF.

56. Certains ingrédients utilisés dans sa fabrication son d'ailleurs envoyés au MET

57. Lettre de Maupoil à Mauss, Athiémé, 22 avril 1935, Collège de France, 57 CDF 8114.

58. Lettre de Maupoil à Rivet, sd, MNHN, 2 AM 1 K64e.

59. Lettre de Lévy-Bruhl à Maupoil, Paris, 13 novembre 1934, CILF.

60. Lettre de Maupoil à Mauss Porto-Novo, 22 août 1934, 57 CDF 81-14 «enquêtes sur le Gbaadu [vodun extrêmement puissant] n'ont pas été fructueuses faute de temps. Les informateurs étaient trop méfiants $\mathbf{5 5}_{\Downarrow}$. Maupoil a fini par obtenir le «secret» du Gbaadu de la part d'un «vieux devin de Nago» indiquant que la description qu'il en fait dans sa thèse est délibérément incomplète:

Au cas où ces lignes viendraient à la connaissance de magiciens noirs du BasDahomey, nous croyons bon de les aviser que nos énumérations sont volontairement incomplètes, et qu'ils ne sauraient se flatter de constituer un Gbaadu avec les seuls éléments dont la liste figure ici. Ceci soit dit dans l'intérêt de leurs ennemis, et pour la paix des tombes $\mathbf{5 6}$ !

(Maupoil 1943: 95)

Une situation d'équilibre, reposant sur des intérêts partagés, doit donc être trouvée pour que l'informateur puisse livrer des informations ou donner des objets, parfois au péril de sa vie ou de sa réputation: bénéficier des «libéralités» accordées par le chercheur, être bien vu par l'administration coloniale, accroître son prestige, valoriser les traditions de Noirs auprès des Blancs, élargir le recrutement des disciples... La confiance, liée en grande partie à la qualité de la relation amicale, et son corollaire, la peur de la trahison, structurent l'ensemble des relations sociales au Dahomey, comme l'a bien étudié Hazoumé dans le LePacte de sang au Dahomey. La rupture du pacte se solde par une punition inéluctable de celui qui n'aurait pas respecté son serment. Or, selon Maupoil, ce «pacte» est mis à mal par la sortie de L'Afrique fantôme. Avec la description de Leiris du vol spectaculaire du Boli, notamment, le sentiment d'obligation de satisfaire aux demandes des colons s'accompagne désormais de la peur d'être trahi:

J'ai lu récemment le livre de Leiris, plein d'une suffisante et enfantine inutilité. Mais surtout, livre susceptible d'incruster la méfiance et l'hostilité dont sont entourées les missions scientifiques africaines. Fort mauvais effet que je déplore vivement57.

Déontologie, parole donnée, croyance..., tout scientifique qu'il est, l'ethnologue est moralement lié à ses informateurs. Au-delà de la qualité de ses travaux, c'est donc également en tant qu'homme qu'il est jugé.

\section{SITUER LE DISCOURS DE L'INFORMATEUR}

Dès son retour de la mission Dakar-Djibouti, dans laquelle il ne se reconnaît pas forcément complètement, Griaule affirme un positionnement ambigu: «l'enquête de sociologie descriptive» devrait, selon lui, primer la collecte d'objets (Griaule 1957: 43-44.). Ainsi, au-delà des nombreux objets envoyés au MET qui, malgré les nombreuses difficultés et certaines zones d'ombre, semblent avoir été acquis selon une ligne déontologique claire, la vraie richesse de la collaboration de Maupoil réside dans la qualité de la documentation qu'il a rassemblée grâce à son terrain prolongé, au réseau d'informateurs qu'il a su constituer et à sa remarquable thèse.

Dans un premier temps, sa position d'administrateur lui permet de nouer des contacts nombreux et privilégiés. Ainsi, il peut dès son arrivée se présenter «aux chefs, aux vieillards et surtout aux veuves

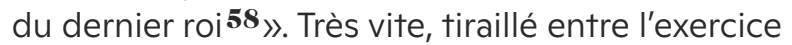
de son activité scientifique et la charge administrative, il est obligé de resserrer son objet d'étude sur le Fa, abandonnant d'autres aspects du vodun (couvents, sociétés masculines, etc.). Rencontrant des difficultés avec sa hiérarchie, Maupoil veut poser sa démission, mais est encouragé à rester, notamment par Lucien Lévy-Bruhl, qui lui rappelle l'opportunité exceptionnelle qui s'offre à lui, confessant à quel point il regrette de ne pas être lui-même sur le terrain:

Je comprends vos impatiences et combien il vous est désagréable d'être tenu par la présence au bureau et la besogne courante, quand il se passe à quelques kilomètres de vous des choses que vous auriez le plus grand désir de voir de vos yeux - qu'aucun autre Blanc peut-être ne se soucie d'observer, et qui ne dureront sans doute plus bien longtemps. Mais, tout de même, vous êtes à pied d'œuvre. Il ya beaucoup que vous pouvez observer, maintenant que vous connaissez les gens et que vous êtes connus $d^{\prime}$ eux $\mathbf{5 9}$.

La définition de son objet d'étude et ses objectifs fluctuent durant la première année de son séjour, sa connaissance des acteurs et sa perception des enjeux religieux et politiques cristallisés autour du Fa étant encore superficielles. Son désir de thèse n'est réellement formulé qu'à l'issue de sa rencontre avec Gèdègbé, qu'il relate dans un courrier adressé à Mauss:

J'ai eu la bonne fortune de trouver, à Abomey même, un bokonon fort savant, qui fut le conseiller intime de Glèlè, et est le chef des bokonon animistes des quatre derniers rois d'Abomey. Il est né vers 1805-1810. Après une prise de contact un peu difficile le premier jour, j'ai vu cet excellent homme se confier peu à peu; quelques jours avant mon départ, il m'a même entretenu de différentes questions politiques du cercle, en m'avouant qu'il violait presque un pacte de sang qui l'unit avec Glèlè en me parlant comme il le faisait. Une sorte d'affection réciproque s'est établie en nous 60. 


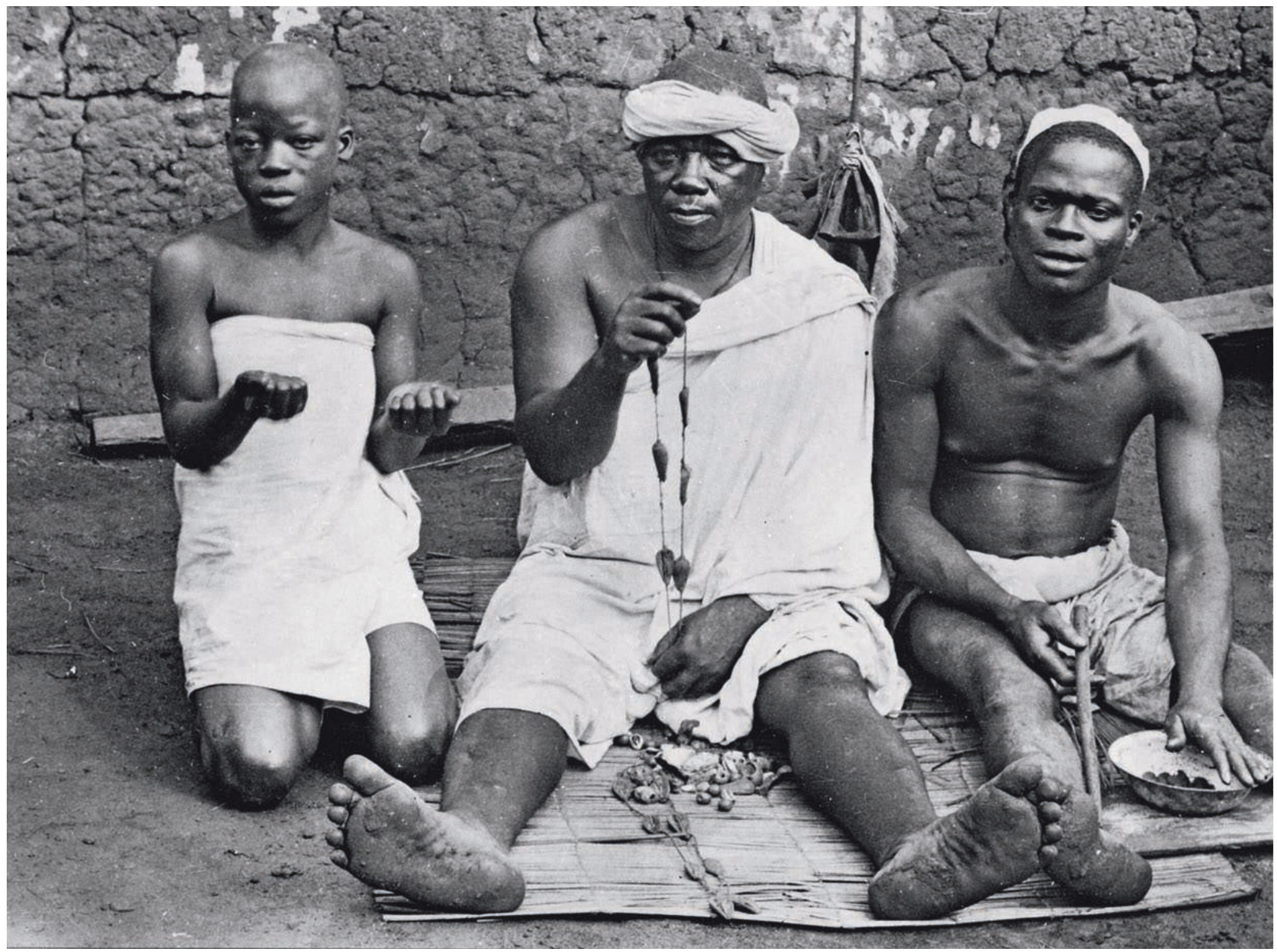

Bernard Maupoil, Consultation de Fa par le Bôkonon à l'aide d'amandes de palme, 1934, tirage sur papier baryté monté sur carton, $22,5 \times 29,5 \mathrm{~cm}$, $n^{\circ}$ inv. PP0036473 (cette photo est présentée dans une vitrine consacrée au Fa en 1973) @ musée du quai Branly-Jacques Chirac.

Mauss plaide en faveur de l'obtention du visa pour la thèse de son élève en saluant le travail de terrain effectué, et la relation exceptionnelle nouée avec Gèdègbé, véritable «trésor humain», dignitaire influant doté d'une réelle capacité réflexive et dernier grand témoin de la période d'avant la colonisation $\mathbf{6 1}$

À cette époque, le rôle de l'informateur se résume généralement à pallier l'absence de sources écrites dans une société orale. Le travail de l'enquêteur consiste à vérifier l'exactitude ce qui est dit, par recoupements successifs. Au départ, Maupoil attribue le manque de cohérence des discours à un éloignement, une dilution du récit «originel». À l'instar des autres ethnologues travaillant sur le vodun, il explique la plasticité des récits par l'absence d'un clergé constitué:

II n'existe pas de chefs de Bokonon qui puissenttrancher un litige, et donner au Blanc cette précieuse assurance que le principe d'identité est sauf $\mathbf{6 2}$.
Très vite cependant, il reconnaît que ces différentes versions sont inhérentes à la situation d'enquête dont elles reflètent la réalité et la richesse. Dépassant les contradictions dues à l'absence de modèle canonique, Maupoil cherche à comprendre l'origine de ces différences et s'interroge sur la possibilité de hiérarchiser leur niveau de pertinence. Au contact de Gèdègbé, il affine sa réflexion et analyse les conditions de transmission des connaissances, qu'il définit finalement comme un véritable «humanisme». Le parcours initiatique du sage dahoméen décrit par Maupoil recoupe celui de l'ethnologue. Les mêmes qualités sont requises: attention, volonté, mémoire, politesse, patience, mais aussi capacité de «choisir des instructeurs compétents $\mathbf{6 3}_{\gg}$. Lorsqu'il distingue les bons et les mauvais informateurs (en particulier les «sorciers»), Maupoil engage son esprit critique et partage sa perception et compréhension des pratiques observées. Contrairement à Griaule, qui apparaît comme un véritable théoricien, notamment lorsqu'il étudie le système de la pensée mythico-poétique des Dogons (Jolly 2001: 174), la thèse de Maupoil est
61. Rapport de Mauss, 11 juillet 1939 , demandant l'accord

du visa pour la thèse, $57 \mathrm{CDF}$ 81-14.

62. Lettre Maupoil à Mauss, 20 juillet 1934, Collège de France, 57 CDF 81-14

63. Note manuscrite et tapuscrite «De l'initiation à l'humanisme», CILF 


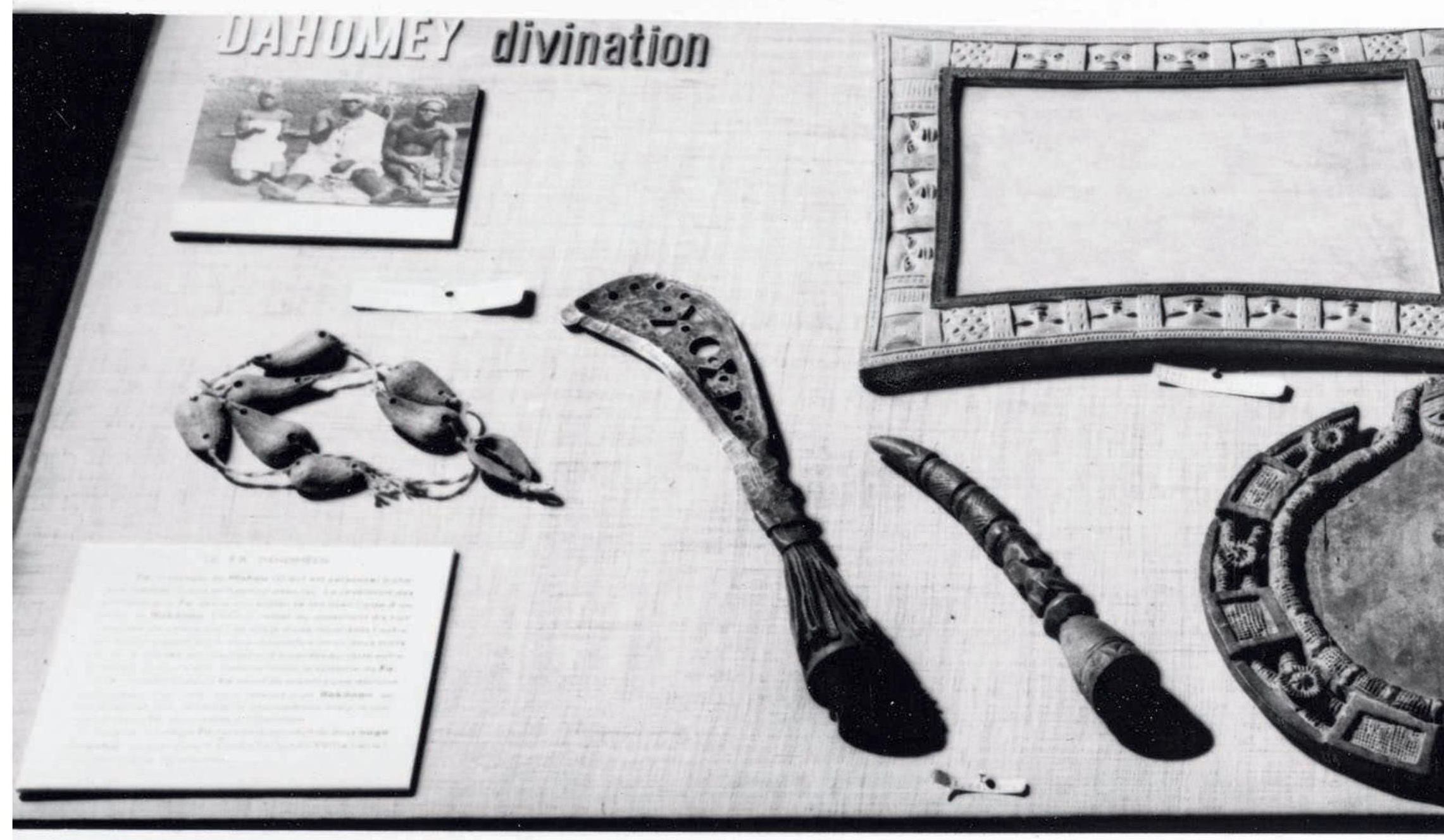

Anonyme, Vitrine 109: Dahomey. Divination. Le fa dahoméen, 1973, tirage sur papier baryté monté sur carton, $22,5 \times 29,5 \mathrm{~cm}, \mathrm{n}^{\circ}$ inv. PP00933775. Cette vitrine comporte une photographie prise par Maupoil (PP0036473) et des objets collectés par ses soins. (c) musée du quai Branly-Jacques Chirac.

64. Maupoil 1988 [1943]: 688 postface de Claude Rivière.

65. Le Pacte de sang

d'Hazoumé est d'abord paru sous forme d'articles dans La Reconnaissance africaine. quasi exempte de démarche interprétative, livrant des faits présentés comme bruts sans réellement dégager de grands principes. Comme l'indique Claude Rivière dans la postface de la réédition de sa thèse, l'originalité de son travail tient à la prise en compte de l'observateur dans la situation d'observation, restituant avec précision les matériaux dans le contexte discursif de l'enquête et s'efforçant d'appréhender les témoignages à l'aune de ce qu'il a compris de la mentalité et des enjeux locaux $\mathbf{6 4}^{\mathbf{4}}$. Son terrain apparaît comme le théâtre d'une expérience intersubjective, où s'élabore la cartographie des informateurs et se racontent les relations nouées avec eux. Ainsi, la correspondance qu'il entretient, notamment avec Melville Herzkovitz - au Dahomey à peu près à la même époque -, donne de la chair aux acteurs rencontrés. À son arrivée, il croise tout d'abord les personnages bien connus d'Aupiais, présents dans ses films, pour la plupart très impliqués dans la gouvernance coloniale. Zounon Medjé d'abord, «le roi de la nuit», figure fantoche et cabotine qui gagne son estime et sa sympathie. Justin Aho ensuite, «l'escroc» qui se sert des étrangers un peu naïfs pour asseoir son prestige politique, avec qui il entre ouvertement en conflit. Ces deux acteurs importants de la scène politique coloniale dahoméenne sont d'ailleurs invités à l'exposition coloniale de 1931, accompagnés par Hazoumé, et séjournent chez Albert à cette occasion par le truchement du père Aupiais.

Maupoil prend assez rapidement ses distances avec ces «notables», figures officielles qui représentent aux yeux des institutions métropolitaines l'autorité politique et religieuse traditionnelle. II noue en revanche une relation plus ambiguë avec les Akawé (terme trouvé dans la correspondance de Maupoil et d'Aupiais), nouvelle classe d'intellectuels «indigènes», initiés à l'ethnographie dans les écoles missionnaires, dont il se sent d'abord proche. Pour Aupiais, les Akawés, qu'il a lui-même en grande partie formés, garantissent la mise en œuvre d'un catholicisme solidement ancré dans le respect des traditions existantes. II les encourage alors à s'intéresser aux richesses culturelles de leur pays, à recueillir, noter contes, légendes, chants, proverbes, coutumes, qu'il fait publier dans la revue la Reconnaissance africaine (Balard 1998: 8365). Encouragé par des membres éminents de l'Institut d'ethnologie, tels qu'Henri Labouret, Aupiais n'a de 
68. Lettre de Maupoil à Mauss 22 novembre 1935, Cotonou, 57 CDF 8114 la réalité de la confrontation entre des subjectivités, des systèmes de valeur, des imaginaires, des sensibilités, mais aussi les efforts qu'il faut faire, de part et d'autre, pour dépasser ces écarts et maintenir une relation. II place ainsi de manière relativement nouvelle les interactions entre observateurs et observés au cœur de la validité scientifique de la recherche empirique (Depraz 1993). Un clin d'œil à I'histoire partagée entre l'ethnologue et son «maître» indigène, placée sous le signe du Fa, vient pourtant discrètement clôturer un chapitre de la thèse, témoignant peut-être d'un glissement hors du champ de la neutralité scientifique:

Les signes Akla-Turukpé et Ce-Abla nous avaient annoncés chez lui, disait-il, alors que nous ne connaissions pas encore son nom; le premier précisa la date de notre rencontre, le second la nature de nos relations.

(Maupoil 1943: 164, n.2)

Quelques mois avant son départ, choqué par la mort de son ami Audric le 7juin 1935, comme lui administrateur et ethnologue, Maupoil confie à Mauss qu'il est probablement condamné lui aussi:

On raconte que lui [Audric] et moi - les deux amis - avons trop «cherché le fond» de ce pays. Audric est mort, et nul ne verra ses papiers. Quantà moi, il m'arrivera aussi quelque chose, et mes papiers disparaîtront [...]. Mais on a déjà faittant de gri-gri contre moi $\mathbf{6 8}$ !!!

Il raconte alors dans sa thèse que Gebegdé lui avait prédit la mort de son compagnon d'aventure. Ce témoignage laisse peu de doute sur l'adhésion du chercheur à la réalité qu'il observe et annonce certainement la génération des ethnologues initiés, notamment incarnée par Pierre Verger pour le Dahomey. Cette posture, qui valorise - comme le proposera Clifford Geertz - la restitution de l'expérience individuelle et permet de rendre poreuses les frontières entre le statut d'observateur et celui d'observé, pourrait parfaitement lui convenir (Geertz 1998). Par sa forte implication, il réussit en effet à dépasser, de manière tout à fait originale, les rapports de domination dans ce contexte colonial au profit d'une recherche a priori plus respectueuse des populations étudiées.

Face à la difficulté de collecter l'expression du sentiment religieux, l'objectif d'une enquête proprement documentaire dans un premier temps (l'objet comme prolongement ou métonymie de la pratique, le texte comme transcription fidèle de la parole entendue, l'image comme miroir du monde visible) se transforme en démarche engagée de la part du chercheur qui oriente ses travaux en fonction de ce qu'il veut démontrer, de ce qu'il ressent et de ce qu'il considère relever de sa légitimité. Comment restituer alors la vérité du terrain, cette «part grise» en tension entre la volonté de prélèvement de fragments de réel et l'irréductible expérience du monde? Selon Leiris, la littérature propose des pistes:

Le sociologue et le psychologue ont beau serrer de plus en plus leurs réseaux de connaissances, toucher de plus en plus près à l'objectivité, ils seront toujours des observateurs, c'est-à-dire situés en pleine subjectivité. Tous les savants en sont là. [...] Un seul homme peut prétendre avoir quelque connaissance de la vie dans ce qui fait sa substance, le poète; parce qu'il se tient au cœur du drame qui se joue entre ces deux pôles: objectivité - subjectivité; parce qu'il les exprime à sa manière qui est le déchirement, dont il se nourrit quant à luimême et dont, quant au monde, il est le porte-venin ou, si l'on veut, porte-parole. (Leiris 1996 [1931] : 450-451, 17 mai 1932, eité par Leborgnne 2017)

Lorsque Maupoil exprime, dans les lettres qu'il envoie à son maître, ses craintes, ses indignations, ses fragilités, sa fascination, son amour immense et souvent douloureux pour ce pays, c'est toute la puissance heuristique du terrain qui transparaît. E† l'on comprend au fil de la lecture, de manière presque intime, comment et à quel point ce temps passé «ailleurs» a ébranlé ses certitudes, a transformé à jamais sa perception du monde, lui a donné la possibilité d'aller au bout de lui-même et l'a finalement transformé - pour un temps hélas trop court - en un véritable ethnologue.

Direction générale des Patrimoines, Ministère de la Culture valerie.perles@culture.gouv.fr 
L'expérience de Bernard Maupoil au Dahomey : entre science et engáagement, un laboratoire pour l'ethnologie en milieu colonial Valérie Perlès

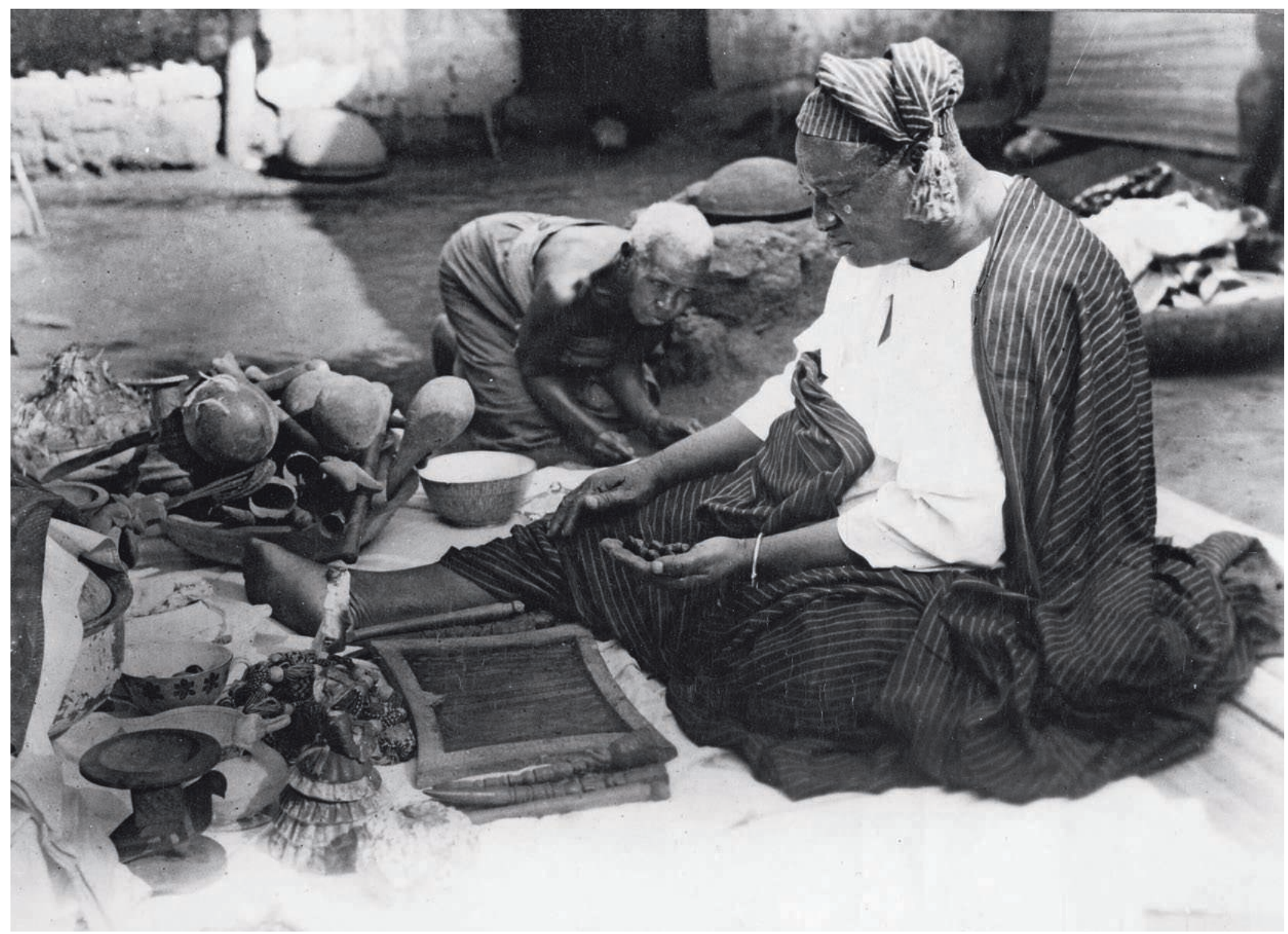

Georges Labitte, Le fils de Gèdègbé consultant l'oracle, 1934-1936?, tirage sur papier baryłé, 22,5 × 29,5cm, $n^{\circ}$ inv. PP0071478 @ musée du quai Branly-Jacques Chirac. 


\section{BIBLIOGRAPHIE}

\section{Amouro, Camille}

2009, «Vodun, bocio et bo», Nanette Jacominj Snoep (dir.) Recettes des dieux; esthétique du fétiche, Musée du Quai Branly, Actes Sud, 2009: 42-43

\section{Balard, Martine}

1996 «Le révérend père Aupiais, artisan d'une reconnaissance africaine », in Beausoleil, Jeanne (dir.), Pour une reconnaissance africaine: Dahomey 1930, des images au service d'une idée: Albert Kahn, 1860-1940 et le père Aupiais, 1877-1945, catalogue d'exposition (19novembre 1996-14septembre 1997). Boulogne-Billancourt, Musée Albert Kahn: 51-78.

1998 Dahomey 1930: mission catholique et culte vodoun. Presse universitaire de Perpignan.

\section{Beaujean-Baltzer, Gaëlle}

2015 "L'Art de Cour à Abomey: le sens des objets. 》 Thèse de doctorat en anthropologie sociale sous la direction de JeanPaul Colleyn et de Henry John Drewal. Paris, EHESS.

Beaujean-Baltzer, Gaëlle et Geoffroy-Schneiter, Bérénice 2009 Artistes d'Abomey : dialogue sur un royaume africain, catalogue d'exposition (10 novembre 2009-31janvier 2010). Paris/ Cotonou, Musée du Quai Branly/ Fondation Zinsou.

\section{Ciarcia, Gaetano}

2019 «Le paganisme et son ordre moral. Le vodun comme "pierre

d'attente" dans le corpus filmique Le Dahomey religieux de Francis Aupiais (1930) $\gg$, Les Carnets de Bérose12 [en ligne], disponible sur: https://www.berose.fr/article1796. html (consulté le 03 janvier 2021).

\section{Conklin, Alice}

2002 «The New "Ethnology" and "la situation coloniale» in Interwar France», French politics, Culture and society 20 (2): 29-45.

2015 «1848-1945: le paradoxe colonial du musée de l'Homme», in Claude Blanckaert (dir.),

Le Musée de l'Homme: histoire

d'un musée laboratoire. Paris, Artlys.

\section{Depraz, Nathalie}

1993 «L'ethnologue, un phénoménologue qui s'ignore? L'apport de la phénoménologie aux sciences sociales», Genèses 10: 108-123.

\section{Dupuis, Annie}

1999 «À propos de souvenirs inédits de Denise Paulme et Michel Leiris: sur la création du musée de l'Homme en 1936 », Cahiers d'Études Africaines 39 (155-156): 511-538.

\section{Geertz, Clifford}

1998 «La description dense. Vers une théorie interprétative de la culture», trad. de l'anglais par André Mary, Enquête 6 [en ligne], disponible sur: https:// journals.openedition.org/ enquete/1443 (consulté le 03 janvier 2021)
Glélé, Maurice Ahanhanzo

1974 Le Danxome: du pouvoir Aja à la nation Fon. Paris, Nubia.

Griaule, Marcel

1957 Méthode de l'ethnographie. Paris, Presses universitaires de France.

\section{Hazoumé, Paul}

1937 Le Pacte de sang au Dahomey. Paris, Institut d'ethnologie.

\section{Hirsch, Thomas}

2017 «I'm the Whole Show. Marcel Mauss professeur à I'Institut d'ethnologie», in André Delpuech, Christine Laurière, Carine Peltier-Caroff (dir.), Les Années folles de l'ethnographie, Trocadéro 28-37. Paris: MNHN 341-402.

\section{Jacomijn Snoep, Nanette}

2009 «Bazar des dieux ou figures de l'informe», in Nanette Jacomijn Snoep (dir.), Recettes des dieux; esthétique du fétiche, catalogue d'exposition (3février-10 mai 2009). Paris/Arles, Musée du Quai Branly/ Actes Sud: 25-34.

\section{Jolly, Éric}

2001 «Marcel Griaule, ethnologue: la construction d'une discipline (1925-1956)», Le Journal des Africanistes 71 (1): 149-190.

Labaune, Christophe

$\mathbf{2 0 1 7}$ «Deux ans en compagnie de Mauss: rétrospective d'une exploration archivistique», Colligere [en ligne], 16 novembre disponible sur: /https://archibibscdf.hypotheses.org/663

(consulté le 14 décembre 2020).

\section{Laurière, Christine}

2008 Paul Rivet, le savant

et le politique. Paris, Publications scientifiques du Muséum national d'histoire naturelle.

2011 «Un américaniste au service de l'ethnologie française. Biographie de Paul Rivet», Bérose - Encyclopédie internationale des histoires de l'anthropologie [en ligne], disponible sur: 10670/1.wbpcue (consulté le 14 décembre 2020).

2017 «L'épreuve du feu des futurs maîtres de l'ethnologie», in A. Delpuech, C. Laurière, $C$. Peltier-Caroff (dir.), Les Années folles de l'ethnographie, Trocadéro 28-37. Paris: MNHN: 405-447.

\section{Leborgne, Érik}

«L'Afrique fantôme de Leiris: un colonialisme unheimlich?», TRANS [en ligne], 17 mars 2017, disponible sur: $\mathrm{http} / / /$ journals. openedition.org/trans/1515 (consulté le 14 décembre 2020).

\section{Leiris, Michel}

2003 [1934] L'Afrique fantôme. Paris, Gallimard.

1996 [1931] L'Afrique fantôme in Miroir de l'Afrique, Jean Jamin (éd.). Paris, Gallimard.
Loyau, Anne

2017 «Le musée d'Ethnographie du Trocadéro et ses transformations, 1878-1935: configurations, espaces muséaux et réseaux», in Arnaud Hurel (dir.), La France savante. CTHS: 234-259.

\section{Maupoil, Bernard}

1943 La Géomancie à l'ancienne Côte des esclaves. Paris, Institut d'ethnologie.

1988 [1943] /dem (3eéd.).

\section{Mauss, Marcel et al.}

1931 Instructions sommaires pour les collecteurs d'objets ethnographiques, musée d'Ethnographie et mission scientifique Dakar-Djibouti. Paris, Palais du Trocadéro.

\section{Thiesse, Anne-Marie}

1996 Les Petites Patries encloses dans la grande: les manuels scolaires régionaux de la III'République. Rapport final à la mission du Patrimoine ethnologique.

\section{Zuccarelli, François}

1973 «De la chefferie traditionnelle au canton: évolution du canton colonial au Sénégal, 1855-1960», Cahiers d'études africaines 13 (50): 213-238.

\section{Archives}

Conseil international de la langue française (CILF), archives Maupoil (en ligne, non cotées).

Collège de France, correspondances reçues Mauss, 57 CDF 81-14 et 57 CFD 108-20.

Muséum national d'histoire naturelle, Bibliothèque centrale, 2 AM 1 K64e. 